\title{
Symbol Theory of Microlocal Operators
}

By

\author{
Keisuke UchIKOSHI
}

\begin{abstract}
Let $u\left(x, x^{\prime}\right) d x^{\prime}$ be an arbitrary microlocal operator. We define the symbol function $\hat{u}(x, \xi)$ of $u\left(x, x^{\prime}\right) d x^{\prime}$, and give a characterization of such symbol functions. Symbol formulae for adjoint operators and for composite operators are given.
\end{abstract}

\section{§ 0. Introduction}

Let $M \subset \boldsymbol{R}^{n}$ be an open set containing the origin, and let $X$ be its complex neighborhood. We identify $M$ with the diagonal set in $M \times M$. The sheaf $\mathscr{L}=\mathscr{L}_{M}$ of microlocal operators is defined on $\sqrt{-1} S^{*} M \cong \sqrt{-1} S_{M}^{*}(M \times M)$ by

$$
\mathscr{L}=\mathscr{H}_{\sqrt{-1} S_{M}^{*}(M \times M)}^{0}\left(\mathscr{C}_{M \times M} \otimes \mathscr{V}_{M}\right)
$$

(See M. Kashiwara and T. Kawai [3] or M. Sato, T. Kawai and M. Kashiwara [11]). Here $\mathscr{C}_{M \times M}$ denotes the sheaf of microfunctions on $M \times M$, and $\mathscr{V}_{M}$ denotes the sheaf of densities on $M$ with real analytic coefficients. A section of $\mathscr{L}$ is called a microlocal operator on $M$, and it acts on microfunctions. This action has the microlocal property, i.e., under this action, the support of each microfunction does not increase.

Roughly speaking, the notion of microlocal operators is the most general one in hyperfunction theory, possessing this microlocal property.

There exist some subclasses of microlocal operators, which are very familiar to us. We denote by $\mathscr{E}^{\infty}=\mathscr{E}_{M}^{\infty}$ (resp. $\mathscr{E}^{R}=\mathscr{E}_{M}^{R}$ ) the sheaf of microdifferential operators (resp. of holomorphic microlocal

Communicated by M. Kashiwara, October 20, 1987. Revised February 22, 1988.

* Department of Mathematics, National Defense Academy, Yokosuka 239, Japan. 
operators) on $M$, defined by [11] (resp. by [4]). Here we do not give their definitions. But later we give an explanation of them from symbol theoretical point of view, and then their meaning will be easily understood. We note that $\mathscr{E}^{\infty} \subset \mathscr{E}^{R} \subset \mathscr{L}$. The symbol theory is already known only for these special subclasses, and our aim is to extend it for general microlocal operators. Only after that, we obtain enough knowledge of them.

To state the main theorem, we give some preliminaries.

Definition 0.1. Let $r>0$ be small. A continuous function $\lambda(t)$ defined on $0<t<r$ is called a scaling function if

$$
\begin{gathered}
\lim _{t \rightarrow+0} \lambda(t) / t=0, \\
t<t^{\prime} \Rightarrow \lambda(t) / t<\lambda\left(t^{\prime}\right) / t^{\prime} .
\end{gathered}
$$

Remark. (i) Let $\lambda(t)$ be a scaling function. It is easy to see that $\lim _{t \rightarrow+0} \lambda(t)=0$, and $t<t^{\prime} \Rightarrow \lambda(t)<\lambda\left(t^{\prime}\right)$. If $\lambda(t)$ is a scaling function, we define $\lambda_{0}(t)$ by $\lambda_{0}(t)=\lambda(t) / t$. If $t=0$, we define $\lambda(t)=\lambda_{0}(t)=0$ 。 It is easy to see that both $\lambda(t)$ and $\lambda_{0}(t)$ are positive definite, and that they always have the inverse functions, $\lambda^{-1}(t)$ and $\lambda_{0}^{-1}(t)$, respectively.

(ii) Taking $r>0$ to be small enough, we may assume that $\lambda(t) / t$ is very small.

Example. (i) $\lambda(t)=m t^{j}, m>0, j>1$.

(ii) $\lambda(t)=m(-\log t)^{j} t, m>0, j>0$.

In this paper, we denote by $x$ the variables in $M$ or $X=M^{c}$, and by $\xi$ the dual variables of $x$.

Now we give the following

Definition 0.2. Let $\stackrel{\circ *}{x}=(0 ; 0, \ldots, 0, \sqrt{-1}) \in \sqrt{-1} S^{*} M$, and let $\lambda(t), \mu(t)$ be two scaling functions. We denote by $\mathscr{S}_{\lambda, \mu}=\left(\mathscr{S}_{\lambda, \mu}\right)_{x^{*}}$ the space of all holomorphic functions $a(x, \xi)$ defined on some conical complex neighborhood $V$ of $\stackrel{\circ}{x}$ such that for any $\varepsilon>0$ there exists some $C_{\varepsilon}>0$ satisfying

(0.3) $|a(x, \xi)| \leqq C_{\varepsilon} \exp \left\{\left(\lambda(|\operatorname{Im} x|)+\mu\left(|\operatorname{Re} \xi| / \operatorname{Im} \xi_{n}\right)+\varepsilon\right) \operatorname{Im} \xi_{n}\right\}$ 
on $V$. We define $\mathscr{S}=\mathscr{S}_{\mathrm{x}^{*}}$ by $\mathscr{S}=\cup_{\lambda, \mu} \mathscr{S}_{\lambda, \mu}$. We denote by $\mathscr{N}=\mathscr{N}_{x^{*}}$ the space of all $a(x, \xi) \in \mathscr{S}$ such that there exist some $\varepsilon>0$ and $C>0$ satisfying

$$
|a(x, \xi)| \leqq C \exp \left(-\varepsilon \operatorname{Im} \xi_{n}\right)
$$

on some conical complex neighborhood of $\stackrel{\circ}{x}^{*}$.

Example. (i) $\exp \left(\sqrt{-1} \xi_{j}^{2} / \xi_{n}\right) \in \mathscr{S}_{{ }^{*}}, 1 \leqq j \leqq n-1$.

(ii) $\exp \left(\sqrt{-1} x_{j}{ }^{2} \xi_{n}\right) \in \mathscr{S}_{{ }^{*}}, 1 \leqq j \leqq n$.

(In $\S 5$ we shall give other examples.)

Let $x, x^{\prime}$ be two points of $M$. Let $u\left(x, x^{\prime}\right) d x^{\prime} \in \mathscr{L}_{x^{*}}$. In $\S 2$ we define the Fourier transform $\hat{u}(x, \xi)$ of $u\left(x, x^{\prime}\right)$ by

$$
\hat{u}(x, \xi)=\int e^{-\left(x-x^{\prime}\right) \xi} u\left(x, x^{\prime}\right) d\left(x-x^{\prime}\right) .
$$

We do not give the precise definition of this integral until $\S 2$, but one may understand that it is naturally defined in the sense of microfunction. Then we have the following

Theorem 0.3. If $u\left(x, x^{\prime}\right) d x^{\prime} \in \mathscr{L}_{0^{*}}$, we have $\hat{u}(x, \xi) \in \mathscr{S}_{x^{*}} / \mathscr{N}_{x^{*}}$, and this Fourier transformation gives an isomorphism

$$
\sigma: \mathscr{L}_{\dot{x}^{*}} \widetilde{\rightarrow} \mathscr{S}_{0^{* *}} / \mathscr{N}_{\dot{x}^{*}}
$$

Definition 0.4. If $A=u\left(x, x^{\prime}\right) d x^{\prime} \in \mathscr{L}_{\text {o }^{*}}$, we call $\sigma(A)=\hat{u}(x, \xi) \in$ $\mathscr{S}_{\mathrm{o}^{*}} / \mathscr{N}_{\mathrm{o}^{*}}$ the symbol function of $A$.

Remark. We denote by $\mathscr{S}_{1}=\left(\mathscr{S}_{1}\right)_{x^{*}}$ the space of all $a(x, \xi) \in \mathscr{S}_{\text {o* }^{*}}$ such that $a(x, \xi)$ satisfy the condition required for $\mathscr{S}_{\text {o }^{*}}$ with $\lambda(t)=$ $\mu(t)=0$ (i.e., the space of infraexponential functions), and by $\mathscr{S}_{2}=$ $\left(\mathscr{S}_{2}\right)_{\alpha^{*}}$ the space of all $a(x, \xi) \in\left(\mathscr{S}_{1}\right)_{\alpha_{*}^{*}}$ which have asymptotic expansions $a(x, \xi) \sim \sum_{j \in Z} a_{j}(x, \xi)$, where each $a_{j}(x, \xi)$ is homogeneous in $\xi$ of degree $j$ (See [11] for the precise definition). In [3] and [11] it is

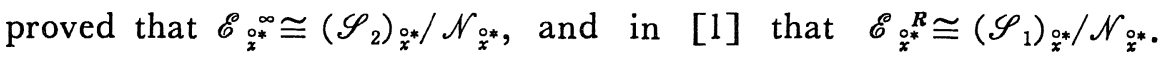
Thus we have

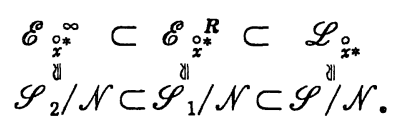


The next results are the symbol formulae for adjoint operators and composite operators of microlocal operators. For that purpose, we need to estimate the derivatives $\partial_{x}^{\alpha} \partial_{\xi}{ }^{\beta} a(x, \xi), \alpha, \beta \in \mathbb{Z}_{+}{ }^{n}$, for $a(x, \xi) \in$ $\mathscr{S}_{\mathrm{o}^{*}}$. Concerning these estimates, it turns out that our symbol space $\mathscr{S}$ has a very similar property as the general symbol space $S_{\rho, \delta}^{m}\left(\mathbb{R}^{n}\right)$, $m \in \mathbb{Z}, 0<\rho, \delta<1$, introduced in distribution theory by L. Hörmander [2]. There exists similarity also to the more general space $S_{\nu, \rho, \delta}^{m}\left(\mathbb{R}^{n}\right)$, $m \in \mathbb{Z}, 0<\rho, \delta<1$, and $\nu(\xi)$ is a "basic weight function", defined by [7]. In $\S 5$ we shall note more precisely that our theory is a generalization of the theory of analytic $S_{\rho, \delta}^{m}$-class defined by $G_{0}$ Métivier [9] (See also [10]). The details will be explained in $\S 3$ and $\S 5$, and here we only note the following fact: Let $a(x, \xi)$ be a symbol function (in distribution theory) belonging to $S_{\rho, \delta}^{m}\left(\mathbb{R}^{n}\right)$. Let $A$ be the corresponding pseudodifferential operator (also in distribution theory), and let $A^{*}$ be its adjoint operator. If $\rho>\delta$, then the symbol of $A^{*}$ is given by the asymptotic expansion

$$
\sigma\left(A^{*}\right)(x,-\xi) \sim \sum_{\alpha} \frac{(-1)^{|\alpha|}}{\alpha !} \partial_{x}^{\alpha} \partial_{\xi}^{\alpha} a(x, \xi) .
$$

However, we cannot expect to obtain such a result if $\rho<\delta$. In fact, if one calculates the above asymptotic expansion formally, each term in (0.5) becomes larger and larger as $|\alpha| \rightarrow+\infty$.

Remark. Let $A=u\left(x, x^{\prime}\right) d x^{\prime} \in \mathscr{L}_{x^{*}}$. In hyperfunction theory, $A^{*}$ is defined by $A^{*}=u\left(x^{\prime}, x\right) d x^{\prime}$. In distribution theory, the same letter $A^{*}$ stands for the complex conjugate of $u\left(x^{\prime}, x\right) d x^{\prime}$. In this paper, we always follow the convention in hyperfunction theory.

Analogous situation occurs in our theory. Let $A \in \mathscr{L}_{\mathbf{x}^{*}}$, and thus the adjoint operator $A^{*}$ belong to $\mathscr{L}_{\dot{x}^{\prime *}}$, where $\stackrel{\circ}{x}^{\prime *}=(0 ; 0, \ldots, 0,-\sqrt{-1})$ $\in \sqrt{-1} S^{*} M$ is the antipodal point of $\stackrel{\circ}{x}^{*}$. Assume that $\sigma(A) \in \mathscr{S}_{\lambda, \mu}$ with some scaling functions $\lambda(t)$ and $\mu(t)$. If one wants to obtain a result as (0.5), one needs to assume the following condition on $\lambda(t)$ and $\mu(t)$, where $C_{0}>0$ and $C_{1}>0$ are regarded as some given constants :

Condition $\mathbb{C}_{0}, \mathbb{C}_{1}$. If $0<t<1 / C_{0}$, then we have $\lambda^{-1}(t) \mu^{-1}(t)>C_{1} t_{\text {。 }}$ 
Example. Let $\lambda(t)=m_{1} t^{i}, \mu(t)=m_{2} t^{j}$, with $m_{1}, m_{2}>0$, and $i, j>1$. Then Condition $C_{0}, C_{1}$ means

$$
\lambda^{-1}(t) \mu^{-1}(t)=\left(t / m_{1}\right)^{1 / i}\left(t / m_{2}\right)^{1 / j}>C_{1} t
$$

and this is satisfied if, and only if, either $1 / i+1 / j<1$ or $1 / i+1 / j=$ $1, m_{1}{ }^{-1 / i} m_{2}{ }^{-1 / j}>C_{1}$. Note that Condition $C_{0}, C_{1}$ should be considered as a condition depending on $C_{0}$ and $C_{1}$.

If $C_{0}, C_{1}$ are large enough, and if $\lambda(t), \mu(t)$ satisfy Condition $C_{0}, C_{1}$, then we have the desired result in the following sense: Let $A=u\left(x, x^{\prime}\right) d x^{\prime} \in \mathscr{L}_{\text {o }^{*}}$ satisfy $\sigma(A)=\hat{u}(x, \xi) \in \mathscr{S}_{\lambda, \mu}$ for these scaling functions. Let $A^{*}=v\left(x, x^{\prime}\right) d x^{\prime}$, and thus $v\left(x, x^{\prime}\right)=u\left(x^{\prime}, x\right)$, by definition. We define $\hat{v}_{j}(x, \xi), j \in Z_{+}=\{0,1,2, \ldots\}$, by

$$
\hat{w}_{j}(x,-\xi)=\sum_{|\alpha|=j} \frac{1}{\alpha !}(-1)^{|\alpha|} \partial_{x}^{\alpha} \partial_{\xi}^{\alpha} \hat{u}(x, \xi),
$$

and define $w_{j}\left(x, x^{\prime}\right), j \in Z_{+}$, by

$$
w_{j}\left(x, x^{\prime}\right)=\frac{1}{(2 \pi \sqrt{-1})^{n}} \int e^{\left(x-x^{\prime}\right) \xi} \hat{w}_{j}(x, \xi) d \xi .
$$

Here each integral is integrated on the domain

$$
\left\{\xi \in \sqrt{-1} R^{n} ; \operatorname{Im} \xi_{n}<-C_{0}\left|\operatorname{Im} \xi_{k}\right|, 1 \leqq k \leqq n-1, \operatorname{Im} \xi_{n}<-C_{0}(j+1)\right\}
$$

for the corresponding number $j \in Z_{+}$. Then we have the following

Theorem 0.5. Let $A \in \mathscr{L}_{\text {o }^{*}}$ satisfy $a(x, \xi)=\sigma(A) \in \mathscr{S}_{\lambda, \mu}$ for some $\lambda$ and $\mu$, and let $\lambda$ and $\mu$ satisfy Condition $C_{0}, C_{1}$ for large $C_{0}$ and $C_{1}$. Let $w_{j}\left(x, x^{\prime}\right), j \in \boldsymbol{Z}_{+}$, be as above. Then $\sum_{j=0}^{\infty} w_{j}\left(x, x^{\prime}\right) d x^{\prime}$ converges and is an element of $\mathscr{L}_{\dot{x}^{\prime *}}$, and we have $A^{*}=\sum_{j=0}^{\infty} w_{j}\left(x, x^{\prime}\right) d x^{\prime}$. In other words, we have the asymptotic formula (0.5).

As for the asymptotic formula for composite operators of microlocal operators, the argument proceeds in a similar way, and we state the following theorem without explaining its precise meaning (See $\S 4$ for the details).

Theorem 0.6. Let $A_{i}=u_{i}\left(x, x^{\prime}\right) d x^{\prime} \in \mathscr{L}_{\text {o }^{*}}, i=1,2$, and assume that 
$\sigma\left(A_{i}\right)=\hat{u}_{i}(x, \xi) \in \mathscr{S}_{\lambda_{i}, \mu_{i}}$ for some scaling function $\lambda_{i}(t)$ and $\mu_{i}(t), i=$ 1, 2. If $\mu_{1}(t)$ and $\lambda_{2}(t)$ satisfy Condition $C_{0}, C_{1}$ with $C_{0}$ and $C_{1}$ large enough, we have

$$
\sigma\left(A_{1} A_{2}\right) \sim \sum_{\alpha} \frac{1}{\alpha !} \partial_{\xi}^{\alpha} \hat{u}_{1}(x, \xi) \partial_{x}^{\alpha} \hat{u}_{2}(x, \xi)
$$

for the composite operator $A_{1} A_{2}$.

Remark. We need to note the relation between our theory and the theory of Fourier integral operators with complex phase functions, due to A. Melin and J.Sjöstrand [8]. Assume that a symbol function $a(x, \xi)$ is of the form

$$
a(x, \xi)=e^{\varphi(x, \xi)} a_{1}(x, \xi) .
$$

Here the amplitude $a_{1}(x, \xi)$ is assumed to be an element of $\mathscr{S}_{1}$, in our notation, and we assume that the phase function $\varphi(x, \xi)$ satisfies $|\operatorname{Re} \varphi(x, \xi)| \leqq\left(\lambda(|\operatorname{Im} x|)+\mu\left(|\operatorname{Re} \xi| / \operatorname{Im} \xi_{n}\right)\right) \operatorname{Im} \xi_{n}$ with some scaling functions $\lambda(t)$ and $\mu(t)$. Then we have $a(x, \xi) \in \mathscr{S}$. On the other hand, $a(x, \xi)$ may be regarded as the symbol of a Fourier integral operator (precisely speaking, a pseudodifferential operator) with a complex phase function. Our theory may be regarded as the generalization of such a theory, since we need not assume that $a(x, \xi)$ is written in the form $(0.7)$. We only need to assume that it satisfies an estimate of the form $(0,3)$.

\section{§1. Preliminaries}

Let $u\left(x, x^{\prime}\right) d x^{\prime}$ be a section of $\mathscr{L}$ defined in a small neighborhood of $x^{*}$. Since we have an identification

$$
\sqrt{-1} S^{*} M \ni(x, \xi) \mapsto(x, \xi ; x,-\xi) \in \sqrt{-1} S_{M}^{*}(M \times M),
$$

$u\left(x, x^{\prime}\right)$ is a section of $\mathscr{C}_{M \times M}$ defined in a small neighborhood of $\left(\stackrel{O}{x}^{*}, \stackrel{\circ}{x}^{\prime *}\right) \in \sqrt{-1} S_{M}^{*}(M \times M)$, whose support is contained in $\sqrt{-1} S_{M}^{*}(M$ $\times M)$. We remind the reader of our notation $\stackrel{\circ}{x}^{*}=(0 ; 0, \ldots, 0, \sqrt{-1})$ and $\stackrel{\circ}{x}^{\prime *}=(0 ; 0, \ldots, 0,-\sqrt{-1})$. In this section, we always assume that

(1.1) for any $x \in M$, supp $u \cap\left(\sqrt{-1} S_{M}^{*}(M \times M)\right)_{x}$ is contained in a small neighborhood of $(\stackrel{\circ}{\xi},-\stackrel{\circ}{\xi})$, where $\stackrel{\circ}{\xi}=(0, \ldots, 0, \sqrt{-1})$. 
Let $v\left(x, x^{\prime}\right)$ be a defining function of $u\left(x, x^{\prime}\right)$. The purpose of this section is to describe under the assumption (1.1) the domain of definition of $v\left(x, x^{\prime}\right)$ in a standard manner, which will be helpful for us later.

Let $a>0$ be a large constant. We introduce a new coordinate system $y=y(x)$ in $M$ by

$$
y_{j}(x)=\left\{\begin{array}{l}
\frac{1}{a} \sum_{k=1}^{n-1} x_{k}-\frac{n}{a} x_{j}+x_{n}, \quad 1 \leqq j \leqq n-1, \\
\frac{1}{a} \sum_{k=1}^{n-1} x_{k}+x_{n}, \quad j=n .
\end{array}\right.
$$

The inverse transformation of $x \mapsto y(x)$ is $x=x(y)$ where

$$
x_{j}(y)=\left\{\begin{array}{l}
\frac{a}{n}\left(-y_{j}+y_{n}\right), \quad 1 \leqq j \leqq n-1, \\
\frac{1}{n} \sum_{k=1}^{n} y_{k}, \quad j=n .
\end{array}\right.
$$

Denoting the dual variables of $x$ (resp. of $y$ ) by $\xi$ (resp. by $\eta$ ), we have

$$
\eta_{j}(\xi)= \begin{cases}-\frac{a}{n} \xi_{j}+\frac{1}{n} \xi_{n}, & 1 \leqq j \leqq n-1, \\ \frac{a}{n} \sum_{k=1}^{n-1} \xi_{k}+\frac{1}{n} \xi_{n}, & j=n,\end{cases}
$$

and

$$
\xi_{j}(\eta)=\left\{\begin{array}{l}
\frac{1}{a} \sum_{k=1}^{n} \eta_{k}-\frac{n}{a} \eta_{j}, \quad 1 \leqq j \leqq n-1, \\
\sum_{k=1}^{n} \eta_{k}, \quad j=n .
\end{array}\right.
$$

Note that we have $x \cdot \xi=x_{1} \xi_{1}+x_{2} \xi_{2}+\ldots+x_{n} \xi_{n}=y(x) \cdot \eta(\xi)$. If $\xi \in$ $\sqrt{-1} R^{n}$ satisfies $\operatorname{Im} \eta_{j}(\xi)>0,1 \leqq j \leqq n$, it follows from (1.4) that $\operatorname{Im} \xi_{n}>\frac{a}{n-1}|\operatorname{Im} \xi|,, 1 \leqq j \leqq n-1$. Since $a>0$ is very large, the first octant $F=\left\{\xi \in \sqrt{-1} R^{n} ; \operatorname{Im} \eta,(\xi)>0,1 \leqq j \leqq n\right\}$ is a small conic neighborhood of $\stackrel{\circ}{\xi}=(0, \ldots, 0, \sqrt{-1})$ in $\sqrt{-1} \boldsymbol{R}^{n}$. Let $G \subset \sqrt{-1} \boldsymbol{R}^{n}$ be another convex cone containing $\stackrel{\circ}{\xi}$, such that $G \Subset F$ but $G$ is very close to $F$. Then $G$ is again a small neighborhood of $\stackrel{\circ}{\xi}$. Our assumption (1.1) may be rewritten in the form 
(1. 1)' $\operatorname{supp} u\left(x, x^{\prime}\right) \subset\left\{(x, \xi ; x,-\xi) \in \sqrt{-1} S_{M}^{*}(M \times M) ; \xi \in G\right\}$, or, equiralently,

(1. 1)" $\quad \operatorname{supp} u\left(\ldots x^{\prime}\right) \subset\left\{\left(x, \xi ; x^{\prime}, \xi^{\prime}\right) \in \sqrt{-1} S_{M}^{*}(M \times M) ; y(x)=y\left(x^{\prime}\right)\right.$, $\left.\eta(\xi)=-\eta\left(\xi^{\prime}\right), \xi \in G\right\}$.

Let $r>0$ be small enoug? and let $\lambda(t)$ be some scaling function. We define $U_{\lambda, r} \subset \boldsymbol{C} \times \boldsymbol{C}$ by

$$
\begin{aligned}
U_{\lambda, r}= & \left\{\left(z, z^{\prime}\right) \in C \times C ; \operatorname{Im}\left(z-z^{\prime}\right)>\lambda(|\operatorname{Im} z|),\right. \\
& |\operatorname{Re} z|<r,|\operatorname{Im} z|<r, \\
& \left.\left|\operatorname{Re}\left(z-z^{\prime}\right)\right|<r,\left|\operatorname{Im}\left(z-z^{\prime}\right)\right|<r\right\},
\end{aligned}
$$

and $U_{\lambda, r}^{(n)} \subset C^{n} \times C^{n}$ by

$$
U_{\lambda, r}^{(n)}=\left\{\left(x, x^{\prime}\right) \in C^{n} \times C^{n} ;\left(y_{j}(x), y_{j}\left(x^{\prime}\right)\right) \in U_{\lambda, r}, 1 \leqq j \leqq n\right\} .
$$

If $\left(x, \xi ; x^{\prime}, \xi^{\prime}\right) \in \operatorname{supp} u\left(x, x^{\prime}\right)$, then from $(1.1)^{\prime \prime}$ we have $\eta(\xi)=$ $-\eta\left(\xi^{\prime}\right)$ and $\xi \in G$. We define $G^{\circ}=\left\{x \in \sqrt{-1} R^{n} ; \operatorname{Im} x \cdot \operatorname{Im} \xi(=\right.$ $\operatorname{Im} y(x) \cdot \operatorname{Im} \eta(\xi)) \geqq 0$ for any $\xi \in G\}$, and $F^{\circ}$ similarly. Then $F^{\circ}=\{x \in$ $\left.\sqrt{-1} R^{n} ; \operatorname{Im} y_{j}(x) \geqq 0,1 \leqq j \leqq n\right\}$, and $G^{\circ}$ is a little larger than $F^{\circ}$. It follows that there exist some $r>0$ and some scaling function $\lambda(t)$ such that $v\left(x, x^{\prime}\right) \in \mathcal{O}\left(U_{\lambda, r}^{(n)}\right)$. Here $\mathcal{O}\left(U_{\lambda, r}^{(n)}\right)$ denotes the space of holomorphic functions on $U_{\lambda, r}^{(n)}$. We describe the situation for the case $n=1$ in figure 1 . Note that if $n=1$, we have $y=x$. The vertical arrow indicates the direction of supp $u\left(x, x^{\prime}\right)$.

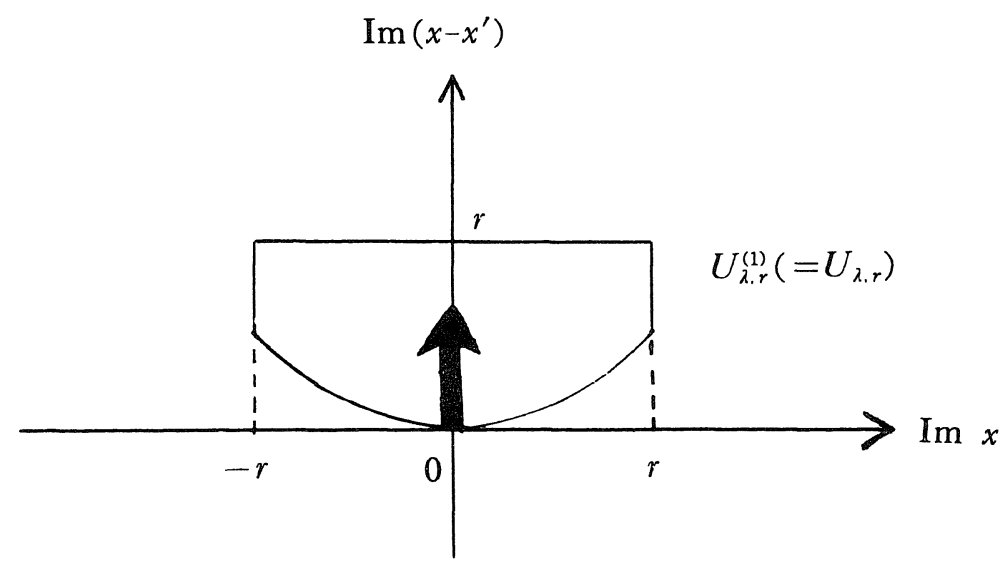

Figure 1 
Assumption (1.1)" means furthermore. supp $u\left(x, x^{\prime}\right)$ is concentrated on the diagonal set $\left\{y(x)=y\left(x^{\prime}\right)\right\}$. Thus $v\left(x, x^{\prime}\right)$ can be continued analytically across the real axis unless $\operatorname{Re} y(x)=\operatorname{Re} y\left(x^{\prime}\right)$. Let $r>0$, and let $\mu(t)$ be a scaling function. We define $V_{\mu, r} \subset \boldsymbol{C} \times \boldsymbol{C}$ by

$$
\begin{aligned}
V_{\mu, r}= & \left\{\left(z, z^{\prime}\right) \in C \times C ;|\operatorname{Im} z|<\mu\left(\left|\operatorname{Re}\left(z-z^{\prime}\right)\right|\right),\right. \\
& \left|\operatorname{Im}\left(z-z^{\prime}\right)\right|<\mu\left(\left|\operatorname{Re}\left(z-z^{\prime}\right)\right|\right) \\
& |\operatorname{Re} z|<r, \quad|\operatorname{Im} z|<r \\
& \left.\left|\operatorname{Re}\left(z-z^{\prime}\right)\right|<r,\left|\operatorname{Im}\left(z-z^{\prime}\right)\right|<r\right\}
\end{aligned}
$$

and $V_{\mu, r}^{(n)} \subset C^{n} \times C^{n}$ by

$$
V_{\mu, r}^{(n)}=\left\{\left(x, x^{\prime}\right) \in C^{n} \times C^{n} ;\left(y,(x), y_{j}\left(x^{\prime}\right)\right) \in V_{\mu, r}, 1 \leqq j \leqq n\right\}
$$

(See Figure 2).

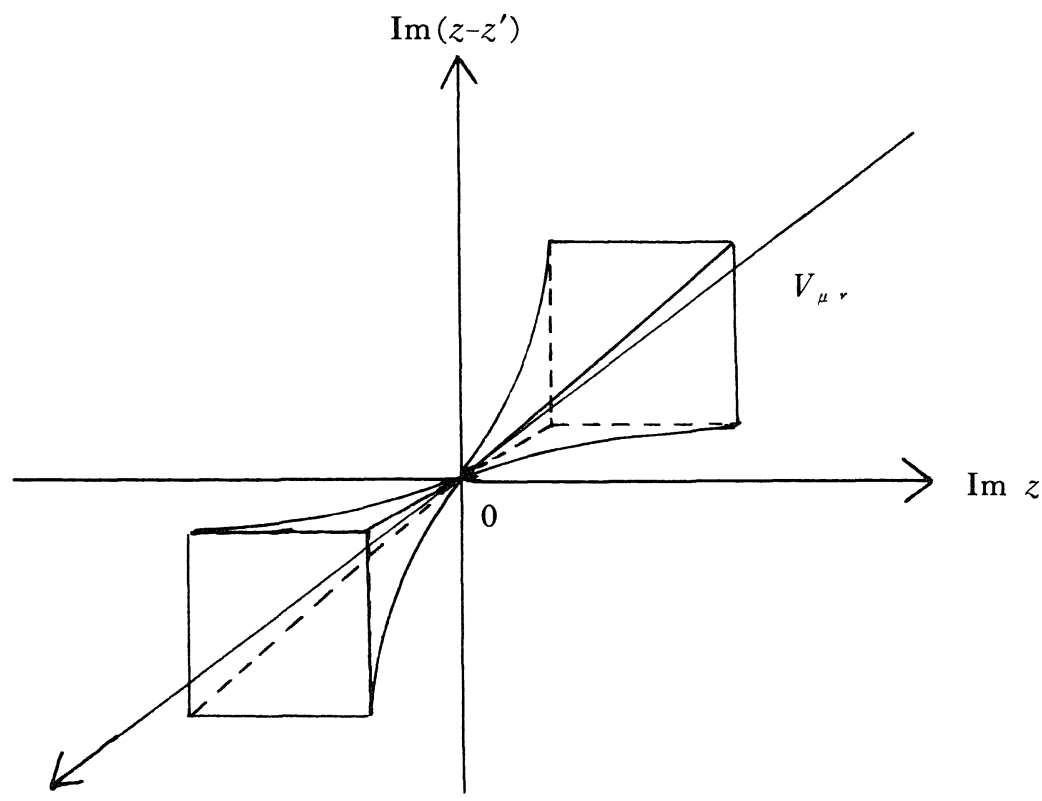

$\operatorname{Re}\left(z-z^{\prime}\right)$

Figure 2 
It is easy to see that if we have chosen $r>0$ small enough, there exist some scaling function $\mu(t)$ such that $v\left(x, x^{\prime}\right) \in \mathcal{O}\left(U_{\lambda, r}^{(n)}\right)$ can be continued analytically to $V_{\mu, r}^{(n)}$.

The advantage of introducing the new coordinate system $y=y(x)$ is that the domain of definition of $v\left(x, x^{\prime}\right)$ can be described in such a direct product form: $U_{\lambda, r}^{(n)} \cup V_{\mu, r}^{(n)} \cong \oplus\left(U_{\lambda, r} \cup V_{\mu, r}\right)$. This will be helpful for us in later arguments. However, this domain $U_{\lambda, r}^{(n)} \cup V_{\mu, r}^{(n)}$ is not very convenient for us, and we need to define the third domain $W_{\lambda^{\prime}, \mu^{\prime}, r^{\prime}}^{(n)}$ as follows.

Let $r^{\prime}>0$, and let $\lambda^{\prime}(t)$ and $\mu^{\prime}(t)$ be two scaling functions. We define $W_{\lambda^{\prime}, \mu^{\prime}, r^{\prime}} \subset \boldsymbol{C} \times \mathbb{C}$ by

$$
\begin{aligned}
& W_{\lambda^{\prime}, \mu^{\prime}, r^{\prime}} \\
= & \left\{\left(z, z^{\prime}\right) \in \mathbb{C} \times \mathbb{C} ; \operatorname{Im}\left(z-z^{\prime}\right)>\lambda^{\prime}(|\operatorname{Im} z|)-\mu^{\prime}\left(\left|\operatorname{Re}\left(z-z^{\prime}\right)\right|\right),\right. \\
& \left.|\operatorname{Re} z|<r^{\prime}, \quad|\operatorname{Im} z|<r^{\prime},\left|\operatorname{Re}\left(z-z^{\prime}\right)\right|<r^{\prime},\left|\operatorname{Im}\left(z-z^{\prime}\right)\right|<r^{\prime}\right\},
\end{aligned}
$$

and $W_{\lambda^{\prime}, \mu^{\prime}, r^{\prime}}^{(n)} \subset \mathbb{C}^{n} \times \mathbb{C}^{n}$ by

$$
W_{\lambda^{\prime}, \mu^{\prime}, r^{\prime}}^{(n)}=\left\{\left(x, x^{\prime}\right) \in \mathbb{C}^{n} \times \mathbb{C}^{n} ;\left(y_{j}(x), y_{j}\left(x^{\prime}\right)\right) \in W_{\lambda^{\prime}, \mu^{\prime}, r^{\prime}}, 1 \leqq j \leqq n\right\} .
$$

The following proposition was suggested by T. Ôaku:

Proposition 1. 1. Let $\lambda^{\prime}(t)=2 \lambda(t), \mu^{\prime}(t)=\lambda(\mu(t))$, and let $r=r^{\prime}$ be small enough. Then we have

$$
W_{\lambda^{\prime}, \mu^{\prime}, r^{\prime}}^{(n)} \subset U_{\lambda, r}^{(n)} \cup V_{\mu, r}^{(n)}
$$

Proof. We remind the reader that we may assume

$$
\lambda(t), \mu(t) \leqq t
$$

(See Remark (ii) after Definition 0.1). Let $\left(z, z^{\prime}\right) \in W_{\lambda^{\prime}, \mu^{\prime}, r^{\prime}}$. We first assume that $|\operatorname{Im} z| \geqq \mu\left(\left|\operatorname{Re}\left(z-z^{\prime}\right)\right|\right)$. Then we have

$$
\begin{aligned}
\operatorname{Im}\left(z-z^{\prime}\right) & >\lambda^{\prime}(|\operatorname{Im} z|)-\mu^{\prime}\left(\left|\operatorname{Re}\left(z-z^{\prime}\right)\right|\right) \\
& =2 \lambda(|\operatorname{Im} z|)-\lambda\left(\mu\left(\left|\operatorname{Re}\left(z-z^{\prime}\right)\right|\right),\right.
\end{aligned}
$$

and from $(1.6)$ it follows that we have $\operatorname{Im}\left(z-z^{\prime}\right)>\lambda(|\operatorname{Im} z|)$, thus $\left(z, z^{\prime}\right) \in U_{\lambda, r}$. We next assume that

$$
|\operatorname{Im} z|<\mu\left(\left|\operatorname{Re}\left(z-z^{\prime}\right)\right|\right) \text {. }
$$

We need to prove that we have either $\operatorname{Im}\left(z-z^{\prime}\right)>\lambda(|\operatorname{Im} z|)$ or $\left|\operatorname{Im}\left(z-z^{\prime}\right)\right|<\mu\left(\left|\operatorname{Re}\left(z-z^{\prime}\right)\right|\right)$. From (1.6) and (1.7), we have $\lambda(|\operatorname{Im} z|) \leqq|\operatorname{Im} z|<\mu\left(\left|\operatorname{Re}\left(z-z^{\prime}\right)\right|\right)$, and thus we only need to prove $\operatorname{Im}\left(z-z^{\prime}\right)>-\mu\left(\left|\operatorname{Re}\left(z-z^{\prime}\right)\right|\right)$. Let $\left(z, z^{\prime}\right) \in W_{\lambda^{\prime}, \mu^{\prime}, r^{\prime}}$, then we 
have

$$
\begin{aligned}
\operatorname{Im}\left(z-z^{\prime}\right) & >\lambda^{\prime}(|\operatorname{Im} z|)-\mu^{\prime}\left(\left|\operatorname{Re}\left(z-z^{\prime}\right)\right|\right) \\
& \geqq-\mu^{\prime}\left(\left|\operatorname{Re}\left(z-z^{\prime}\right)\right|\right)=-\lambda\left(\mu\left(\left|\operatorname{Re}\left(z-z^{\prime}\right)\right|\right)\right),
\end{aligned}
$$

and from (1.6) it follows that $\operatorname{Im}\left(z-z^{\prime}\right)>-\mu\left(\left|\operatorname{Re}\left(z-z^{\prime}\right)\right|\right)$.

Q. E. D.

Let $\kappa(t)$ and $\kappa^{\prime}(t)$ be two scaling functions. We say that $\kappa(t)$ and $\kappa^{\prime}(t)$ have the same order if there exist some constants $m_{1}$ and $m_{2}$ such that $\kappa(t)=m_{1} \kappa^{\prime}\left(m_{2} t\right)$. In Proposition 1. $1, \lambda(t)$ and $\lambda^{\prime}(t)$ have the same order, but $\mu(t)$ and $\mu^{\prime}(t)$ do not. This may be regarded as defect. In fact, the orders of the scaling functions need not be changed:

Proposition 1.2. Let $\lambda^{\prime}(t)=2 \lambda(8 t), \mu^{\prime}(t)=\frac{1}{2} \mu\left(\frac{1}{5} t\right)$, and $0<r^{\prime}$ $\ll r$. Then any holomorphic function defined on $U_{\lambda, r}^{(n)} \cup V_{\mu, r}^{(n)}$ can be continued analytically to $W_{\lambda^{\prime}, \mu^{\prime}, r^{\prime}}^{(n)}$

Proposition 1.2 has advantage to Proposition 1.1 form the above point of view. But whether one uses Proposition 1.1 or Proposition 1.2, the conclusion is the same, in the whole context of this section: There exist some $r>0$, and some scaling functions $\lambda(t), \mu(t)$, such that $v\left(x, x^{\prime}\right) \in \mathcal{O}\left(W_{\lambda, \mu, r}^{(n)}\right)$. Thus from logical point of view, Proposition 1.2 may be regarded as unnecessary, although it has its own interest. We do not give the proof of Proposition 1.2 by this reason, and only note that it follows from a local version of Bochner's tube theorem, due to M. Kashiwara and H. Komatsu [6].

\section{§2. Fourier Transformation of Microlocal Operators}

In this section we define the Fourier transform $\hat{u}(x, \xi)$ of $u(x$, $\left.x^{\prime}\right) d x^{\prime} \in \mathscr{L}_{x^{*} \text {. }}$ In this section, again we assume that $u\left(x, x^{\prime}\right)$ satisfies the assumption (1.1), for the moment. The general case will be treated at the end of this section. Let $v\left(x, x^{\prime}\right)$ be a defining function of $u\left(x, x^{\prime}\right)$. We fix some $a>0$, and define $y=y(x)$ by (1.2), as before, and thus we have $v\left(x, x^{\prime}\right) \in \mathcal{O}\left(W_{\lambda, \mu, r}^{(n)}\right)$, for some $\lambda(t), \mu(t)$, and $r$. We define $W_{r} \subset C \times C$ by

$$
W_{r}=\left\{\left(z, z^{\prime}\right) \in \boldsymbol{C} \times \boldsymbol{C} ;|\operatorname{Re} z|<r, \quad|\operatorname{Im} z|<r,\right.
$$




$$
\left.\left|\operatorname{Re}\left(z-z^{\prime}\right)\right|<r,\left|\operatorname{Im}\left(z-z^{\prime}\right)\right|<r\right\} \text { 。 }
$$

We have $W_{\lambda, \mu, r}=\left\{\left(z, z^{\prime}\right) \in W_{r} ; \operatorname{Im}\left(z-z^{\prime}\right)>\lambda(|\operatorname{Im} z|)-\mu(\mid \operatorname{Re}(z-\right.$ $\left.\left.\left.z^{\prime}\right) \mid\right)\right\}$, and $W_{\lambda, \mu, r}^{(n)} \cong \oplus W_{\lambda, \mu, r}$. We define $\partial_{k} W_{\lambda, \mu, r, \varepsilon_{0}}^{(n)}, 1 \leqq k \leqq n, \varepsilon_{0}>0$, by

$$
\begin{aligned}
\partial_{k} W_{\lambda, \mu, r, \varepsilon_{0}}^{(n)}= & \left\{\left(x, x^{\prime}\right) \in \mathbb{C}^{n} \times \mathbb{C}^{n} ;\left(y_{j}(x), y_{j}\left(x^{\prime}\right)\right) \in W_{\lambda, \mu, r}, j \neq k,\right. \\
& \operatorname{Im}\left(y_{k}(x)-y_{k}\left(x^{\prime}\right)\right)>\lambda\left(\left|\operatorname{Im} y_{k}(x)\right|\right) \\
& \left.-\mu\left(\left|\operatorname{Re}\left(y_{k}(x)-y_{k}\left(x^{\prime}\right)\right)\right|\right)-\varepsilon_{0},\left(y_{k}(x), y_{k}\left(x^{\prime}\right)\right) \in W_{r}\right\} .
\end{aligned}
$$

We define $\mathcal{O}\left(\partial_{k} W_{\lambda, \mu, r}^{(n)}\right)=\underset{\varepsilon_{0}>0}{\bigcup} \mathcal{O}\left(\partial_{k} W_{\lambda, \mu, r, \varepsilon_{0}}^{(n)}\right)$. Note that if $v\left(x, x^{\prime}\right) \in$ $\bigoplus_{k=1}^{n} \mathcal{O}\left(\partial_{k} W_{\lambda, \mu, r}^{(n)}\right)$, then $\left(\stackrel{\circ *}{x^{*}}, \stackrel{\circ}{x}^{*}\right) \notin \operatorname{supp} u\left(x, x^{\prime}\right)$, and thus the corresponding microlocal operator is zero at ${ }^{*}$

Inspired by [1] and [5], we define $\hat{u}(x, \xi)$ as follows: Let $s_{1}, s_{2}$ $\in \mathbb{C}$ be such that

$$
\left\{\begin{array}{l}
-r<\operatorname{Re} s_{1}<0,-r<\operatorname{Im} s_{1}<0 \\
0<\operatorname{Re} s_{2}<r,-r<\operatorname{Im} s_{2}<0
\end{array}\right.
$$

Let $r^{\prime}>0$ be small enough $\left(r^{\prime} \ll r\right)$. We assume that $s_{1}$ and $s_{2}$ satisfy

$$
\operatorname{Im} s_{i}>\lambda\left(r^{\prime}\right)-\mu\left(\left|\operatorname{Re} s_{i}\right|\right), i=1,2 .
$$

It is easy to see that if $z \in \mathbb{C}$ satisfies $|\operatorname{Re} z|<r^{\prime},|\operatorname{Im} z|<r^{\prime}$, then we have $\left(z, z-s_{i}\right) \in W_{\lambda, \mu, r}, i=1,2$. Let $C>0$ be large enough. Then we have

(2.4) $\operatorname{Im} s_{i}<-\frac{1}{C} \mu\left(\left|\operatorname{Re} s_{i}\right|\right)$.

Now we can define $\hat{u}(x, \xi)$ :

Definition 2.1. We define the Fourier transform $\hat{u}(x, \xi)$ of $u\left(x, x^{\prime}\right)$ by

(2.5) $\hat{u}(x, \xi)=\hat{v}(x, \xi)=\int_{\Gamma} e^{-\left(x-x^{\prime}\right) \xi_{v}}\left(x, x^{\prime}\right) d\left(x-x^{\prime}\right)$.

Here $\Gamma=\left\{x-x^{\prime} \in C^{n} ; y_{3}(x)-y_{j}\left(x^{\prime}\right) \in r, 1 \leqq j \leqq n\right\}$, and $\gamma$ is a continuous curve from $s_{1}$ to $s_{2}$.

Remark. (i) From now on, we write as $y_{j}=y_{j}(x), y_{j}{ }^{\prime}=y_{j}\left(x^{\prime}\right)$, and $\eta_{j}=\eta_{,}(\xi)$. If confusion is not likely, we write as $v\left(x, x^{\prime}\right)=$ $v\left(y, y^{\prime}\right)$, by abuse of words. Since we have $\partial\left(x-x^{\prime}\right) / \partial\left(y-y^{\prime}\right)=$ $(-n / a)^{n-1},(2.5)$ may be rewritten as

$(2.5)^{\prime} \hat{u}(x, \xi)=\hat{v}(x, \xi)$ 


$$
=\left(\frac{-n}{a}\right)^{n-1} \int_{s_{1}}^{s_{2}} \ldots \int_{s_{1}}^{s_{2}} e^{-\left(y-y^{\prime}\right) \eta_{v}} v\left(y, y^{\prime}\right) d\left(y_{1}-y_{1}^{\prime}\right) \ldots d\left(y_{n}-y_{n}{ }^{\prime}\right) .
$$

(ii) Definition 2.1 has some ambiguity. This definition depends on the choice of the defining function $v\left(x, x^{\prime}\right)$, on the choice of the new coordinate system $y=y(x)$, and on the choice of $s_{1}, s_{2}$. It turns out that this does not matter, but we do not discuss about it for the moment.

Let $x \in C^{n}$ satisfy

(2.6) $\left|\operatorname{Re} y_{j}\right|<r^{\prime},\left|\operatorname{Im} y_{j}\right|<r^{\prime}, 1 \leqq j \leqq n$.

For any $\varepsilon>0$, we can choose $\gamma=\gamma_{\varepsilon}$ as follows: If $x \in C^{n}$ satisfies (2.6) and $y_{j}-y_{j}{ }^{\prime} \in \gamma_{\varepsilon}, 1 \leqq j \leqq n$, then we have $\left(x, x^{\prime}\right) \in W_{\lambda_{\mu}, r}^{(n)}$ and

(2.7) $\operatorname{Im}\left(y_{j}-y_{j}{ }^{\prime}\right)<\lambda\left(\left|\operatorname{Im} y_{j}\right|\right)-\frac{1}{C} \mu\left(\left|\operatorname{Re}\left(y_{j}-y_{j}{ }^{\prime}\right)\right|\right)+\varepsilon, 1 \leqq j \leqq n$.

This is clear from figure 3 , where $A$ denotes the curve $\operatorname{Im}\left(y_{j}-y_{j}{ }^{\prime}\right)$ $=\lambda\left(\left|\operatorname{Im} y_{j}\right|\right)-\mu\left(\left|\operatorname{Re}\left(y_{j}-y_{j}{ }^{\prime}\right)\right|\right)$, and $B$ denotes $\operatorname{Im}\left(y_{j}-y_{j}{ }^{\prime}\right)=$ $\lambda\left(\left|\operatorname{Im} y_{j}\right|\right)-\frac{1}{C} \mu\left(\left|\operatorname{Re}\left(y_{j}-y_{j}{ }^{\prime}\right)\right|\right)+\varepsilon$. Let us fix $x \in C^{n}$ which satisfies (2.6). If each $y_{j}-y_{j}{ }^{\prime}$ belongs to the shaded domain, we have $\left(x, x^{\prime}\right) \in$

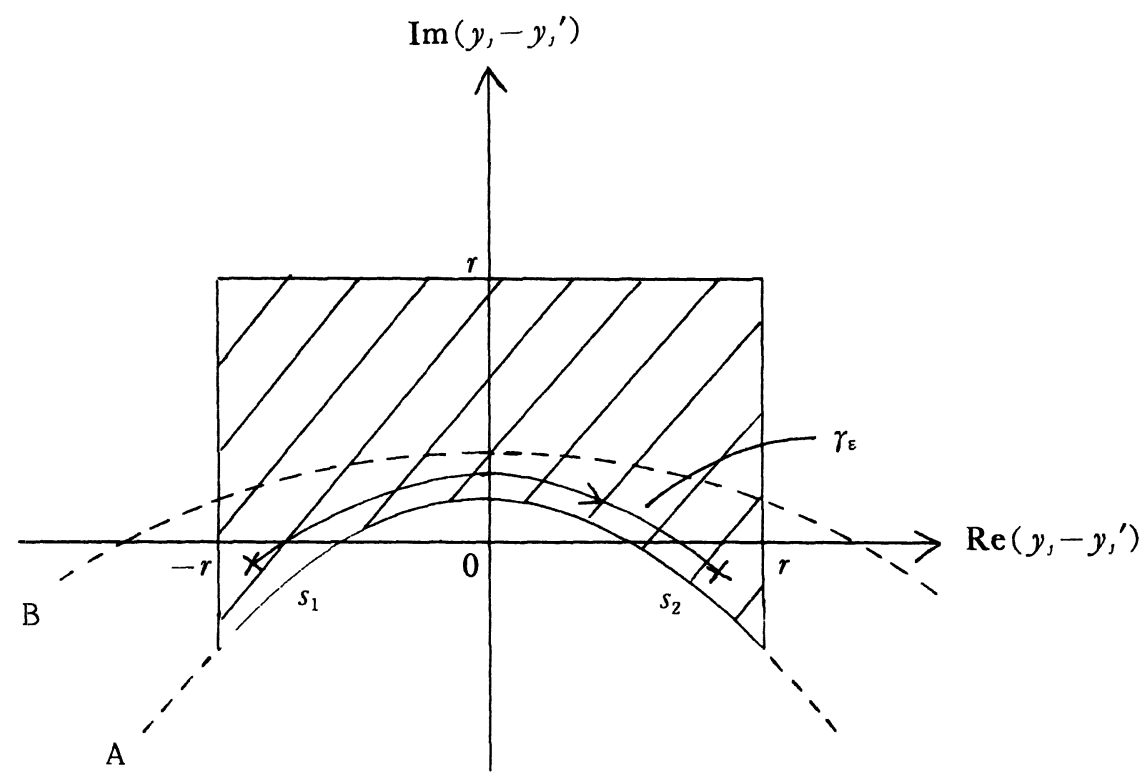

Figure 3 
$W_{\lambda, \mu, r}^{(n)}$, and it is easy to see that (2.7) holds.

Now we define $T_{r} \subset C^{n} \times \mathbb{C}^{n}, r>0$, by

$$
\begin{gathered}
T_{r}=\left\{(x, \xi) \in C^{n} \times C^{n} ;\left|\operatorname{Re} y_{j}\right|<r, \quad\left|\operatorname{Im} y_{j}\right|<r,\right. \\
\left.r \operatorname{Im} \eta_{j}>\left|\operatorname{Re} \eta_{j}\right|, r \operatorname{Im} \eta_{j}>1, \quad 1 \leqq j \leqq n\right\}
\end{gathered}
$$

Now we give two definitions.

Definition 2.2. Let $r>0$, and let $\lambda(t), \mu(t)$ be two scaling functions. We define $\mathcal{O}_{\lambda, \mu}\left(T_{r}\right)$ by

$$
\begin{aligned}
\mathcal{O}_{\lambda, \mu}\left(T_{r}\right)= & \left\{a(x, \xi) \in \mathcal{O}\left(T_{r}\right) ; \text { for any } \varepsilon>0,\right. \text { there exists some } \\
& C_{\varepsilon}>0 \text { such that }|a(x, \xi)| \leqq C_{\varepsilon} \exp \left\{\sum _ { j = 1 } ^ { n } \left(\lambda\left(\left|\operatorname{Im} y_{j}\right|\right)\right.\right. \\
& \left.\left.\left.+\mu\left(\left|\operatorname{Re} \eta_{j}\right| / \operatorname{Im} \eta_{j}\right)+\varepsilon\right) \times \operatorname{Im} \eta_{j}\right\} \text { on } T_{r}\right\} .
\end{aligned}
$$

We define $\partial_{k} \mathcal{O}_{\lambda, \mu}\left(T_{r}\right), 1 \leqq k \leqq n$, by

$$
\begin{aligned}
\partial_{k} \mathcal{O}_{\lambda, \mu}\left(T_{r}\right)= & \left\{a(x, \xi) \in \mathcal{O}\left(T_{r}\right) ; \text { there exists some } \varepsilon_{0}>0\right. \text { and for } \\
& \text { any } \varepsilon>0 \text { there exists some } C_{\varepsilon}>0 \text { such that } \mid \\
& a(x, \xi) \mid \leqq C_{\varepsilon} \exp \left\{\sum _ { j = 1 } ^ { n } \left(\lambda\left(\left|\operatorname{Im} y_{j}\right|\right)+\mu\left(\left|\operatorname{Re} \eta_{j}\right| / \operatorname{Im} \eta_{j}\right)\right.\right. \\
& \left.\left.+\varepsilon) \operatorname{Im} \eta_{j}-\varepsilon_{0} \operatorname{Im} \eta_{k}\right\} \text { on } T_{r}\right\} .
\end{aligned}
$$

Definition 2.3. Let $\lambda(t)=t \lambda_{0}(t)$ be a scaling function. We define its dual scaling function $\tilde{\lambda}(t)$ by $\tilde{\lambda}(t)=t \lambda_{0}^{-1}(t)$.

Example. If $\lambda(t)=m t^{i}, m>0, i>1$, then we have $\bar{\lambda}(t)=m^{-1 /(i-1)}$ $t^{i /(i-1)}$.

Lemma 2.4. (i) $\tilde{\lambda}(t)$ is also a scaling function.

(ii) The dual function $\bar{\lambda}(t)$ of $\tilde{\lambda}(t)$ coincides with $\lambda(t)$.

The proof of Lemma 2.4 is easy. Now we have the following

Proposition 2.5. Let $0<r^{\prime} \ll r, C \gg 1$, and let $\mu^{\prime}(t)=\tilde{\mu}(C t)$. Then we have

(i) If $v\left(x, x^{\prime}\right) \in \mathcal{O}\left(W_{\lambda, \mu, r}^{(n)}\right)$, then $v(x, \xi) \in \mathcal{O}_{\lambda, \mu^{\prime}}\left(T_{r^{\prime}}\right)$.

(ii) If $v\left(x, x^{\prime}\right) \in \mathcal{O}\left(\partial_{k} W_{\lambda, \mu, r}^{(n)}\right)$, then $\hat{v}(x, \xi) \in \partial_{k} \mathcal{O}_{\lambda, \mu^{\prime}}\left(T_{r^{\prime}}\right)$.

Proof. Let $v\left(x, x^{\prime}\right) \in \mathcal{O}\left(W_{\lambda, \mu, r}^{(n)}\right)$. For any $\varepsilon>0$ small, we define $\gamma_{\varepsilon}$ as above. Let $(x, \xi) \in T_{r^{\prime}}$, and let $y_{j}-y_{j}^{\prime} \in \gamma_{\varepsilon}, 1 \leqq j \leqq n$. Taking $r^{\prime}$ small, we may assume that $\operatorname{Im} \eta_{j}>C\left|\operatorname{Re} \eta_{j}\right|, 1 \leqq j \leqq n$. From (2.7) we 
have

(2.8)

$$
\begin{aligned}
& \left|e^{-\left(y-y^{\prime}\right) \eta_{v}}\left(y, y^{\prime}\right)\right| \\
\leqq & C_{\varepsilon} \exp \left\{\sum_{j=1}^{n} \operatorname{Im}\left(y_{j}-y_{j}{ }^{\prime}\right) \operatorname{Im} \eta_{j}+\sum_{j=1}^{n}\left|\operatorname{Re}\left(y_{j}-y_{j}{ }^{\prime}\right)\right| \cdot\left|\operatorname{Re} \eta_{j}\right|\right\} \\
\leqq & C_{\varepsilon} \exp \left\{\sum_{j=1}^{n}\left(\lambda\left(\left|\operatorname{Im} y_{j}\right|\right)+\varepsilon\right) \operatorname{Im} \eta_{j}\right. \\
& \left.\quad+\sum_{j=1}^{n}\left(-\frac{1}{C} \mu\left(\left|\operatorname{Re}\left(y_{j}-y_{j}^{\prime}\right)\right|\right) \operatorname{Im} \eta_{j}+\left|\operatorname{Re}\left(y_{j}-y_{j}^{\prime}\right)\right| \cdot\left|\operatorname{Re} \eta_{j}\right|\right)\right\} .
\end{aligned}
$$

We can prove

(2.9) $I=-\frac{1}{C} \mu\left(\left|\operatorname{Re}\left(y_{j}-y_{j}{ }^{\prime}\right)\right|\right) \operatorname{Im} \eta_{j}+\left|\operatorname{Re}\left(y_{j}-y_{j}{ }^{\prime}\right)\right| \cdot\left|\operatorname{Re} \eta_{j}\right|$

$$
\leqq \tilde{\mu}\left(C\left|\operatorname{Re} \eta_{j}\right| / \operatorname{Im} \eta_{j}\right) \operatorname{Im} \eta_{j}
$$

as follows: If $\left|\operatorname{Re}\left(y_{j}-y_{j}{ }^{\prime}\right)\right| \geqq \mu_{0}{ }^{-1}\left(C\left|\operatorname{Re} \eta_{j}\right| / \operatorname{Im} \eta_{j}\right)$, then

$$
\begin{aligned}
I= & -\frac{1}{C}\left|\operatorname{Re}\left(y_{j}-y_{j}^{\prime}\right)\right| \mu_{0}\left(\left|\operatorname{Re}\left(y_{j}-y_{j}^{\prime}\right)\right|\right) \operatorname{Im} \eta_{j} \\
& +\left|\operatorname{Re}\left(y_{j}-y_{j}^{\prime}\right)\right| \cdot\left|\operatorname{Re} \eta_{j}\right| \\
\leqq & -\frac{1}{C}\left|\operatorname{Re}\left(y_{j}-y_{j}^{\prime}\right)\right|\left(C\left|\operatorname{Re} \eta_{j}\right| / \operatorname{Im} \eta_{j}\right) \operatorname{Im} \eta_{j} \\
& +\left|\operatorname{Re}\left(y_{j}-y_{j}^{\prime}\right)\right| \cdot\left|\operatorname{Re} \eta_{j}\right|=0 .
\end{aligned}
$$

Contrarily, if $\left|\operatorname{Re}\left(y_{j}-y_{j}{ }^{\prime}\right)\right| \leqq \mu_{0}{ }^{-1}\left(C\left|\operatorname{Re} \eta_{j}\right| / \operatorname{Im} \eta_{j}\right)$, then

$$
\begin{aligned}
I & \leqq\left|\operatorname{Re}\left(y_{j}-y_{j}{ }^{\prime}\right)\right| \cdot\left|\operatorname{Re} \eta_{j}\right|=\mu_{0}^{-1}\left(C\left|\operatorname{Re} \eta_{j}\right| / \operatorname{Im} \eta_{j}\right)\left|\operatorname{Re} \eta_{j}\right| \\
& \leqq \tilde{\mu}\left(C\left|\operatorname{Re} \eta_{j}\right| / \operatorname{Im} \eta_{j}\right) \operatorname{Im} \eta_{j},
\end{aligned}
$$

From (2.8) and (2.9), we have

$$
\begin{aligned}
& \left|e^{-\left(y-y^{\prime}\right) \eta_{v}}\left(y, y^{\prime}\right)\right| \\
\leqq & C_{\varepsilon} \exp \left\{\sum_{j=1}^{n}\left(\lambda\left(\left|\operatorname{Im} y_{\jmath}\right|\right)+\tilde{\mu}\left(C\left|\operatorname{Re} \eta_{\jmath}\right| / \operatorname{Im} \eta_{\jmath}\right)+\varepsilon\right) \operatorname{Im} \eta_{\jmath}\right\},
\end{aligned}
$$

which proves (i). To prove (ii), let $v\left(x, x^{\prime}\right) \in \mathcal{O}\left(\partial_{k} W_{\lambda, \mu, r}^{(n)}\right)$. We can take the path $\Gamma$ in (2.5) as follows: If $x-x^{\prime} \in \Gamma$, then $\operatorname{Im}\left(y_{k}\right.$ $\left.-y_{k}{ }^{\prime}\right)<\lambda\left(\left|\operatorname{Im} y_{k}\right|\right)-\frac{1}{C} \mu\left(\left|\operatorname{Re}\left(y_{k}-y_{k}{ }^{\prime}\right)\right|\right)-\varepsilon_{0}$, and for $j \neq k,\left(y_{j}, y_{j}{ }^{\prime}\right)$ is as above. Then the proof is the same as above. $\quad$ Q.E.D.

We next show the converse result. Let $a(x, \xi) \in \mathcal{O}_{\lambda, \mu}\left(T_{r}\right)$, and define $\check{a}\left(x, x^{\prime}\right)$ by

$$
\check{a}\left(x, x^{\prime}\right)=\frac{1}{(2 \pi \sqrt{-1})^{n}} \int_{\Delta} e^{\left(x-x^{\prime}\right) \xi} a(x, \xi) d \xi,
$$

where $\Delta=\left\{\xi \in C^{n} ; \eta=\eta(\xi) \in \sqrt{-1} \boldsymbol{R}_{+}{ }^{n} \operatorname{Im} \eta_{j}>1 / r, 1 \leqq j \leqq n\right\}$. The domain of integration $(2.10)$ is thus a small conical neighborhood of $(0, \ldots, 0, \sqrt{-1})$ in $\sqrt{-1} R^{n}$, in the original coordinate system. Then 
we have the following

Proposition 2. 6. Let $\mu^{\prime}(t)=\tilde{\mu}(t / 2)$, and let $0<r^{\prime} \ll r$. Then we have

(i) If $a(x, \xi) \in \mathcal{O}_{\lambda, \mu}\left(T_{r}\right)$, then $\check{a}\left(x, x^{\prime}\right) \in \mathcal{O}\left(W_{\lambda, \mu^{\prime}, r^{\prime}}^{(n)}\right)$.

(ii) If $a(x, \xi) \in \partial_{k} \mathcal{O}_{\lambda, \mu}\left(T_{r}\right)$, then $\check{a}\left(x, x^{\prime}\right) \in \mathcal{O}\left(\partial_{k} W_{\lambda, \mu^{\prime}, r^{\prime}}^{(n)}\right)$.

Proof. Let $a(x, \xi) \in \mathcal{O}_{\lambda, \mu}\left(T_{r}\right)$, and let $\left(x, x^{\prime}\right) \in W_{\lambda, \mu^{\prime}, r^{\prime}}^{(n)}$ (i.e., $\left(y_{j}, y_{j}{ }^{\prime}\right)$ $\in W_{\left.\lambda, \mu^{\prime}, r^{\prime}, 1 \leqq j \leqq n\right) . ~ L e t ~} J_{i}, J_{2} \subset\{1,2, \ldots, n\}$ be such that $J_{1} \cup J_{2}=$ $\{1,2, \ldots, n\}$ is a disjoint union. We define $J=\left(J_{1}, J_{2}\right)$. Let $J$ be such a pair, and we assume that $\left(x, x^{\prime}\right)$ satisfies $\operatorname{Re}\left(y_{j}-y_{j}{ }^{\prime}\right) \geqq 0$ (resp. $<0)$ if $j \in J_{1}\left(\operatorname{resp} . J_{2}\right)$. It is easy to see that

$(2.10)^{\prime} \quad \check{a}\left(x, x^{\prime}\right)=\frac{1}{(2 \pi \sqrt{-1})^{n}} \int_{\Delta_{J}} e^{\left(x-x^{\prime}\right) \xi} a(x, \xi) d \xi$,

where

$$
\begin{aligned}
\Delta_{J}= & \left\{\xi \in C^{n} ; \operatorname{Im} \eta_{j}>1 / r, 1 \leqq j \leqq n,\right. \\
& \operatorname{Re} \eta_{j}=-\mu_{0}^{-1}\left(\left|\operatorname{Re}\left(y_{j}-y_{j}^{\prime}\right)\right| / 2\right)\left(\operatorname{Im} \eta_{j}-1 / r\right), j \in J_{1}, \\
& \left.\operatorname{Re} \eta_{j}=\mu_{0}^{-1}\left(\operatorname{Re}\left(y_{j}-y_{j}^{\prime}\right) \mid / 2\right)\left(\operatorname{Im} \eta_{j}-1 / r\right), j \in J_{2}\right\} .
\end{aligned}
$$

Then for any $\varepsilon>0$ there exists some $C_{\varepsilon}>0$ such that

$$
\begin{aligned}
&\left|e^{\left(x-x^{\prime}\right) \xi} a(x, \xi)\right|=\left|e^{\left(y-y^{\prime}\right) \eta} a(y, \eta)\right| \\
& \leqq C_{\varepsilon} \exp \left\{-\sum_{j=1}^{n} \operatorname{Im}\left(y_{j}-y_{j}{ }^{\prime}\right) \operatorname{Im} \eta_{j}+\sum_{j=1}^{n} \operatorname{Re}\left(y_{j}-y_{j}^{\prime}\right) \operatorname{Re} \eta_{j}\right. \\
&\left.\quad+\sum_{j=1}^{n}\left(\lambda\left(\left|\operatorname{Im} y_{j}\right|\right)+\mu\left(\left|\operatorname{Re} \eta_{j}\right| / \operatorname{Im} \eta_{j}\right)+\varepsilon\right) \operatorname{Im} \eta_{j}\right\},
\end{aligned}
$$

if $\xi \in \Delta_{J}$. Note that

(2. 12) $\operatorname{Re}\left(y_{j}-y_{j}{ }^{\prime}\right) \operatorname{Re} \eta_{j}=-2 \tilde{\mu}\left(\left|\operatorname{Re}\left(y_{j}-y_{j}{ }^{\prime}\right)\right| / 2\right)\left(\operatorname{Im} \eta_{j}-1 / r\right)$

and

(2. 13) $\mu\left(\left|\operatorname{Re} \eta_{j}\right| / \operatorname{Im} \eta_{j}\right)=\mu_{0}\left(\left|\operatorname{Re} \eta_{j}\right| / \operatorname{Im} \eta_{j}\right)\left|\operatorname{Re} \eta_{j}\right| / \operatorname{Im} \eta_{j}$

$$
\begin{aligned}
& \leqq\left(\left|\operatorname{Re}\left(y_{j}-y_{j}{ }^{\prime}\right)\right| / 2\right) \mu_{0}{ }^{-1}\left(\left|\operatorname{Re}\left(y_{j}-y_{j}{ }^{\prime}\right)\right| / 2\right) \\
& =\tilde{\mu}\left(\left|\operatorname{Re}\left(y_{j}-y_{j}{ }^{\prime}\right)\right| / 2\right) .
\end{aligned}
$$

From $(2.11)-(2.13)$ it follows that

$$
\begin{aligned}
& \left|e^{\left(x-x^{\prime}\right) \xi} a(x, \xi)\right| \\
& \leqq C_{\varepsilon} \exp \left\{-\sum_{j=1}^{n}\left(\operatorname{Im}\left(\gamma_{j}-y_{j}{ }^{\prime}\right)-\lambda\left(\left|\operatorname{Im} y_{j}\right|\right)+\tilde{\mu}\left(\left|\operatorname{Re}\left(y_{j}-y_{j}{ }^{\prime}\right)\right| / 2\right)\right.\right. \\
& \left.\quad-\varepsilon) \operatorname{Im} \eta_{j}\right\} .
\end{aligned}
$$

Since $J$ and $\varepsilon$ are arbitrary, the integral (2.10)' defines an element of $\mathcal{O}\left(W_{\lambda, \mu^{\prime}, r^{\prime}}^{(n)}\right)$. This proves (i), and the proof of (ii) is similar. 
Q. E. D.

We next consider the relation of the above two propositions.

Definition 2.7. Let $\lambda(t)$ be a scaling function. We denote by [ $\lambda]$ the set of all scaling functions which have the same order as $\lambda$, i.e., $[\lambda]=\left\{\lambda^{\prime}(t)=m_{1} \lambda\left(m_{2} t\right) ; m_{1}, m_{2}>0\right\}$. We define

$$
\begin{aligned}
& \mathcal{O}\left(W_{[\lambda],[\mu]}^{(n)}\right)=\bigcup_{\lambda^{\prime} \in[\lambda]} \underset{\mu^{\prime} \in[\mu]}{\cup} \underset{r>0}{\cup} \mathcal{O}\left(W_{\lambda^{\prime}, \mu^{\prime}, r^{\prime}}^{(n)},\right. \\
& \mathcal{O}[\lambda],[\mu]=\bigcup_{\lambda^{\prime} \in[\lambda]} \bigcup_{\mu^{\prime} \in[\mu]}^{\cup} \bigcup_{r>0} \mathcal{O}_{\lambda^{\prime}, \mu^{\prime}}\left(T_{r}\right),
\end{aligned}
$$

and $\mathcal{O}\left(\partial_{k} W_{[\lambda],[\mu]}^{(n)}\right), \partial_{k} \mathcal{O}_{[\lambda],[\mu]}, 1 \leqq k \leqq n$, similarly.

Let $w\left(x, x^{\prime}\right) \in \mathcal{O}\left(W_{[\lambda],[\mu]}^{(n)}\right) / \bigoplus_{k=1}^{n} \mathcal{O}\left(\partial_{k} W_{[\lambda],[\mu]}^{(n)}\right)$. We have $w\left(x, x^{\prime}\right) \equiv$ $v\left(x, x^{\prime}\right)$ modulo $\bigoplus_{k=1}^{n}\left(\partial_{k} W_{[\lambda],[\mu]}^{(n)}\right)$ with some $v\left(x, x^{\prime}\right) \in \mathcal{O}\left(W_{\lambda^{\prime}, \mu^{\prime}, r}^{(n)}\right)$, $\lambda^{\prime} \in[\lambda], \mu^{\prime} \in[\mu]$, and $r>0$. Note that $\hat{v}(x, \xi) \in \mathcal{O}_{[\lambda],[\tilde{\mu}]} / \bigoplus_{k} \partial_{k} \mathcal{O}_{[\lambda],[\bar{\mu}]}$ is defined independently of the choice of $s_{1}$ and $s_{2}$. In fact, assume that we have replaced $s_{1}$ and $s_{2}$ by another pair $s_{1}^{\prime}$ and $s_{2}^{\prime}$, and let $\hat{v}^{\prime}(x, \xi)$ be the correspondiug Fourier transform of $v\left(x, x^{\prime}\right)$. It is

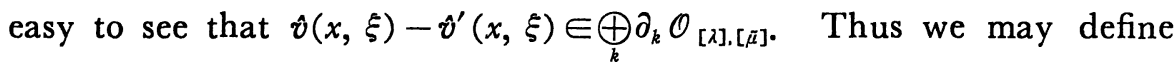
$\hat{w}(x, \xi) \in \mathcal{O}_{[\lambda],[\bar{\mu}]} / \bigoplus_{k} \partial_{k} \mathcal{O}_{[\lambda],[\bar{\mu}]}$ by $\hat{w}(x, \xi)=\hat{v}(x, \xi)$. Conversely let $b(x, \xi) \in \mathcal{O}_{[\lambda],[\tilde{\mu}]} / \bigoplus_{k} \partial_{k} \mathcal{O}_{[\lambda],[\tilde{\mu}]}$ and let $b(x, \xi) \equiv a(x, \xi)$ modulo $\bigoplus_{k} \partial_{k}$ $\mathcal{O}_{[\lambda] .[\tilde{\mu}]}$ with some $a(x, \xi) \in \mathcal{O}_{\lambda^{\prime \prime}, \mu^{\prime \prime}}\left(T_{r^{\prime}}\right), \lambda^{\prime \prime} \in[\lambda], \mu^{\prime \prime} \in[\tilde{\mu}]$, and $r^{\prime}>0$. It is easy to see that we can define $\check{b}\left(x, x^{\prime}\right) \in \mathcal{O}\left(W_{[\lambda] .[\tilde{\mu}]}^{(n)}\right) / \bigoplus_{k} \mathcal{O}\left(\partial_{k} W_{[\lambda] .[\tilde{\mu}]}^{(n)}\right)$ by $\check{b}\left(x, x^{\prime}\right)=\check{a}\left(x, x^{\prime}\right)$. Since $\tilde{\mu}(t)=\mu(t)$, we have defined the following two maps:

$$
\frac{\mathcal{O}\left(W_{[\lambda],[\mu]}^{(n)}\right)}{\bigoplus_{k} \mathcal{O}\left(\partial_{k} W_{[\lambda],[\mu]}^{(n)}\right)} \stackrel{\widehat{\hookrightarrow}}{\longleftarrow} \frac{\mathcal{O}_{[\lambda],[\mu]}}{\bigoplus_{k} \partial_{k} \mathcal{O}_{[\lambda],[\mu]}}
$$

We have the following

\section{Proposition 2. 8. (2.14) is an isomorphism.}

Proof. We need to prove the following:

(i) Let $v\left(x, x^{\prime}\right) \in \mathcal{O}\left(W_{\lambda^{\prime}, \mu^{\prime}, r}^{(n)}\right)$ with $\lambda^{\prime} \in[\lambda], \mu^{\prime} \in[\mu], r>0$, and $a(x, \xi)=\hat{v}(x, \xi)$. Then we have $\check{a}\left(x, x^{\prime}\right)-v\left(x, x^{\prime}\right) \in \bigoplus_{k} \mathcal{O}\left(\partial_{k}\right.$ $\left.W_{[\lambda],[\tilde{\mu}]}^{(n)}\right)$.

(ii) Let $a(x, \xi) \in \mathcal{O}_{\lambda^{\prime}, \mu^{\prime}}\left(T_{r}\right)$ with $\lambda^{\prime} \in[\lambda], \quad \mu^{\prime} \in[\mu], r>0$, 


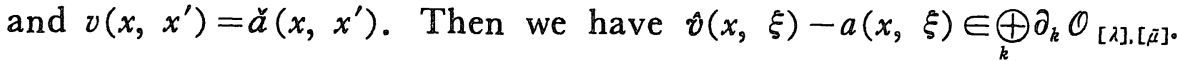

Both statements are proved in a standard manner, and we only give a sketch of the proof of (i). Let $0<r^{\prime} \ll r$, and assume that $\left|\operatorname{Im} y_{j}\right|<r^{\prime} / 2, \operatorname{Im}\left(y_{j}-y_{j}{ }^{\prime}\right)>r^{\prime} / 2,1 \leqq j \leqq n$. With the above notation we have

$$
\begin{aligned}
& \check{a}\left(x, x^{\prime}\right)=\frac{1}{(2 \pi \sqrt{-1})^{n}} \int_{\Delta} \int_{\Gamma} e^{\left(x^{\prime}-x^{\prime \prime}\right) \xi} v\left(x, x^{\prime \prime}\right) d\left(x-x^{\prime \prime}\right) d \xi \\
& =\frac{1}{(2 \pi \sqrt{-1})^{n}} \int_{\Gamma} \int_{\Delta} e^{\left(x^{\prime}-x^{\prime \prime} \xi_{v}\right.}\left(x, x^{\prime \prime}\right) d \xi d\left(x-x^{\prime \prime}\right) \\
& =\frac{1}{(2 \pi \sqrt{-1})^{n}} \int_{s_{1}}^{s_{2}} \ldots \int_{s_{1}}^{s_{2}} \int_{\sqrt{-1 / r}}^{\sqrt{-1} \infty} \cdots \int_{\sqrt{-1 / r}}^{\sqrt{-1} \infty} \\
& \times e^{\left(y^{\prime}-y^{\prime \prime \prime}\right)} v\left(y, y^{\prime \prime}\right) d \eta d\left(y-y^{\prime \prime}\right) \\
& =\frac{1}{(2 \pi \sqrt{-1})^{n}} \int_{s_{1}}^{s_{2}} \ldots \int_{s_{1}}^{s_{2}} \prod_{j=1}^{n}\left(y_{j}^{\prime \prime}-y_{j}^{\prime}\right)^{-1} e^{\sqrt{-1}\left(y_{j}^{\prime}-y_{j}^{\prime \prime}\right) / r} \\
& \times v\left(y, y^{\prime \prime}\right) d\left(y-y^{\prime \prime}\right) \\
& \equiv v\left(y, y^{\prime}\right)=v\left(x, x^{\prime}\right) \text { modulo } \underset{k}{\oplus} \mathcal{O}\left(\partial_{k} W_{[1],[\mu]}^{[n]}\right) . Q \mathbb{E} . \mathbb{D} \text {. }
\end{aligned}
$$

Now we show that Definition 2.1 has no ambiguity. We first remind the reader that we have already noted that this definition does not depend on the choice of $s_{1}$ and $s_{2}$.

From Definition 0.2, Definition 2.2, and Definition 2.7, it is easy to see that we have $\mathcal{O}_{[\{],[\mu]} \subset \mathscr{S}_{x}$, and $\bigoplus_{k} \partial_{k} \mathcal{O}_{[\lambda],[\mu]} \subset \mathscr{N}_{x}$, and thus we may consider $\hat{v}(x, \xi)$ as an element of $\mathscr{S}_{x^{\circ}} / \mathscr{N}_{\AA^{\circ}}$. Now we show that $\hat{v}(x, \xi) \in \mathscr{S}_{x^{\circ}} / \mathscr{N}_{x^{*}}$ is defined with no ambiguity。 $\hat{v}(x, \xi)$ is independent of the choice of the new coordinate system $y=y(x)$ defined by (2.2). To prove this, we first note that we have found that without loss of generality we may choose $s_{1}, s_{2}$ as $s_{1}=-r / 2-$ $\sqrt{-1} r^{\prime}, s_{2}=r / 2-\sqrt{-1} r^{\prime}, 0<r^{\prime} \ll r$. In the integral $(2.5)^{\prime}$, each path from $s_{1}$ to $s_{2}$ may be replaced by

$$
\begin{aligned}
\left\{y_{j}-y_{j}{ }^{\prime} \in \mathbb{C} ;\right. & \left|\operatorname{Re}\left(y_{j}-y_{j}{ }^{\prime}\right)\right|<r / 2, \\
& \left.\operatorname{Im}\left(y_{j}-y_{j}{ }^{\prime}\right)=\varphi\left(\max _{i}\left|\operatorname{Re}\left(y_{i}-y_{i}{ }^{\prime}\right)\right|\right)\right\},
\end{aligned}
$$

where

$$
\varphi(t)= \begin{cases}-r^{\prime}, & t \geqq r / 4, \\ r / 2-\left(2 r+4 r^{\prime}\right) t / r, & 0 \leqq t \leqq r / 4 .\end{cases}
$$

In the original coordinate system $(x, \xi)$, this means that in the 
integral (2.5) the domain of integration $\Gamma$ may be replaced by

$$
\begin{aligned}
\left\{x-x^{\prime} \in C^{n} ;\right. & \left|\operatorname{Re}\left(y_{j}(x)-y_{j}\left(x^{\prime}\right)\right)\right|<r / 2,1 \leqq j \leqq n, \\
& \operatorname{Im}\left(x_{j}-x_{j}{ }^{\prime}\right)=0,1 \leqq j \leqq n-1, \\
& \left.\operatorname{Im}\left(x_{n}-x_{n}{ }^{\prime}\right)=\varphi\left(\max _{i}\left|\operatorname{Re}\left(y_{i}(x)-y_{i}\left(x^{\prime}\right)\right)\right|\right)\right\} .
\end{aligned}
$$

Neglecting an element of $\mathscr{N}_{x^{*}}$, this domain can be replaced by

$$
\begin{aligned}
\left\{x-x^{\prime} \in C^{n} ;\right. & \left|\operatorname{Re}\left(x_{j}-x_{j}{ }^{\prime}\right)\right|<r / 2, \quad 1 \leqq j \leqq n, \\
& \operatorname{Im}\left(x_{j}-x_{j}{ }^{\prime}\right)=0,1 \leqq j \leqq n-1, \\
& \left.\operatorname{Im}\left(x_{n}-x_{n}{ }^{\prime}\right)=\varphi\left(\max _{i}\left|\operatorname{Re}\left(x_{i}-x_{i}{ }^{\prime}\right)\right|\right)\right\},
\end{aligned}
$$

which does not depend on $y=y(x)$.

Assume that $u\left(x, x^{\prime}\right) \in \mathscr{H}_{\sqrt{-1} s_{M}^{0}}^{*(M \times M)}\left(\mathscr{C}_{M \times M}\right)$ satisfies (1.1). Then the definition of $\hat{u}(x, \xi)$ does not depend on the choice of a defining function of $u\left(x, x^{\prime}\right)$. In fact $v\left(x, x^{\prime}\right) \in \mathcal{O}\left(W_{\lambda, \mu, r}^{(n)}\right)$ which defines $u\left(x, x^{\prime}\right)$ is uniquely determined by the injectivity of the boundary operator.

We finally consider the case without the assumption (1.1). Let $u\left(x, x^{\prime}\right) \in \mathscr{H}_{\sqrt{-1} S_{M}^{*}(M \times M)}^{0}\left(\mathscr{C}_{M \times M}\right)$. Let $\omega \subset \sqrt{-1} S^{2 n-1}$ be a small neighborhood of $(\stackrel{\circ}{\xi},-\stackrel{\circ}{\xi})$, and $u\left(x, x^{\prime}\right)$ be defined for $\left(\xi, \xi^{\prime}\right) \in \omega$. Let $\omega^{\prime} \Subset$ $\omega$ be another neighborhood of $\stackrel{\circ}{(\xi,},-\stackrel{\circ}{\xi})$. We choose $u_{1}\left(x, x^{\prime}\right) \in$ $\mathscr{H}_{\sqrt{-1}}^{0} S_{*^{(M \times M)}}\left(\mathscr{C}_{M \times M}\right)$ such that $u_{1}\left(x, x^{\prime}\right)=0$ if $\left(\xi, \xi^{\prime}\right) \notin \omega$ and $u_{1}\left(x, x^{\prime}\right)$ $=u\left(x, x^{\prime}\right)$ if $\left(\xi, \xi^{\prime}\right) \in \omega^{\prime}$. We define $\hat{u}(x, \xi) \in \mathscr{S}_{0_{*}^{*}} / \mathscr{N}_{x}$ by $\hat{u}(x, \xi)=$ $\hat{u}_{1}(x, \xi)$. To prove that this is well-defined, we need to show that if $u_{1}\left(x, x^{\prime}\right)$ satisfies $(1.1)$ and $\left(\stackrel{\circ *}{x}, \stackrel{\circ}{x}^{*}\right) \notin \operatorname{supp} u_{1}$, then $\hat{u}_{1}(x, \xi) \in \mathscr{N}_{x}$. We can easily prove this fact, using the microanalyticity of $u_{1}\left(x, x^{\prime}\right)$. In fact, in (2.5) we can choose the path of integration $\Gamma$ in such a way that $\hat{u}_{1}(x, \xi)$ becomes exponentially decreasing at $\stackrel{\circ}{*}^{*}$, and thus $\hat{u}_{1}(x, \xi) \in \mathscr{N}_{x}$. Thus we have obtained the following map:

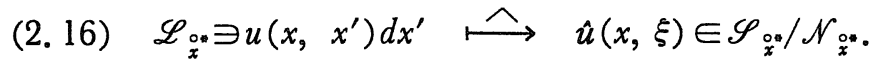

To prove that this is an isomorphism, we first prepare the following

Proposition 2.9. Let $r>0$ be small enough. There exist some $C>0$ and a holomorphic function $e(\eta)$ defined on $\left\{\eta \in C^{n} ; r \operatorname{Im} \eta_{j}>\left|\operatorname{Re} \eta_{j}\right|\right.$, $1 \leqq j \leqq n\}$, which satisfy the following : 
(i) We have

$$
|e(\eta)| \leqq C|\operatorname{Im} \eta|^{c} \exp \left\{C \sum_{j=1}^{n}\left|\operatorname{Re} \eta_{j}\right|^{2} / \operatorname{Im} \eta_{j}\right\}
$$

on the above domain of definition.

(ii) If $2 \operatorname{Im} \eta_{j}>\operatorname{Im} \eta_{k}$ for any $j$ and $k$ furthermore, we have

$$
|e(\eta)-1| \leqq C \exp \left\{-\frac{1}{C} \sum_{j=1}^{n} \operatorname{Im} \eta_{j}\right\} \text {. }
$$

(iii) If $\operatorname{Im} \eta_{j}>C \operatorname{Im} \eta_{k}$ for some $j$ and $k$ furthermore, we have

$$
|e(\eta)| \leqq C \exp \left\{-\frac{1}{C} \sum_{j=1}^{n} \operatorname{Im} \eta_{j}\right\}
$$

Before the proof, we explain the meaning of this proposition. The above statement (i) says that $e(\xi)=e(\eta)$ defines a microlocal operator $e(D)$ at $\stackrel{\circ}{x}^{*}$ (This follows from Proposition 2.8). From (ii) it follows that $e(D)$ is equivalent to the identity operator near $\eta_{1}=$ $\eta_{2}=\ldots=\eta_{n}$, i.e. near $\stackrel{\circ}{x}^{*}$ in $(x, \xi)$ variables, and from (iii) it follows that $e(D)$ is equivalent to the zero operators near the boundary of the first octant. Thus $e(D)$ may be regarded as an analytic partition of unity.

Now we give the sketch of the proof of Proposition 2.9. We first assume that $n=2$. We define $\Phi(y)=\Phi\left(y_{1}, y_{2}\right)$ by

$$
\Phi(y)=\frac{2}{(2 \pi \sqrt{-1})^{2}} \prod_{j=1}^{2}\left(y_{j}+\sum_{k=1}^{2}\left(\frac{y_{k}}{2}+\sqrt{-1} y_{k}^{2}\right)\right)^{-1}
$$

We take the boundary value of $\Phi(y)$ from $\left\{\operatorname{Im} y_{j}>0, j=1,2\right\}$, and obtain the corresponding microfunction $[\Phi(y)]$. It is easy to see that

$$
\begin{gathered}
\operatorname{supp}[\Phi(y)] \subset\left\{(y, \eta) \in \sqrt{-1} T^{*} \boldsymbol{R}^{2} ; y=0, \operatorname{Im} \eta_{j}<3 \operatorname{Im} \eta_{k}, 1 \leqq j, k \leqq 2\right\} \\
\subset\left\{(y, \eta) \in \sqrt{-1} T^{*} \boldsymbol{R}^{2} ; y=0, \operatorname{Im} \eta_{j}>0, j=1,2\right\} .
\end{gathered}
$$

We can define $e(\eta)=\hat{\Phi}(\eta)$ by

$$
e(\eta)=\int e^{-y \eta} \Phi(y) d y
$$

just in the same way as (2.5), and calculating this integral in the same way as there, we obtain (i). Since $[\Phi(y)]$ is equivalent to the delta function near $\stackrel{\circ}{x}^{*}$, we obtain (ii). Lastly, since $\operatorname{supp}[\Phi(y)]$ is completely contained in the first octant, we obtain (iii). And we can prove Proposition 2.9 in this way if $n=2$.

We next consider the case for general $n$. Let us denote the above 
$e\left(\eta_{1}, \eta_{2}\right)$ by $e_{0}\left(\eta_{1}, \eta_{2}\right)$, and let us define $e\left(\eta_{1}, \ldots, \eta_{n}\right)$ by

$$
e\left(\eta_{1}, \ldots, \eta_{n}\right)=\prod_{1 \leqq j<k \leqq n} e_{0}\left(\eta_{3}, \eta_{k}\right) \text {. }
$$

This function satisfies the above requirements.

Now let us prove that (2.16) is an isomorphism, using Proposition 2.9. Let $b(x, \xi) \in \mathscr{S}_{x}$. Let $a>0$ be large enough, and define $y=y(x), \eta=\eta(\xi)$ by (1.2), (1.4). Then there exists some scaling functions $\lambda(t), \mu(t)$, and for any $\varepsilon>0$ there exists some $C_{\varepsilon}>0$ such that

(2. 17) $|b(x, \xi)| \leqq C_{\varepsilon} \exp \left\{\sum_{j=1}^{n}\left(\lambda(|\operatorname{Im} y|)+\mu\left(\left|\operatorname{Re} \eta_{j}\right| / \operatorname{Im} \eta_{j}\right)+\varepsilon\right)\right.$ $\left.\times \operatorname{Im} \eta_{j}\right\}$.

(2.17) is not sufficient for $b(x, \xi) \in \mathcal{O}_{\lambda, \mu}\left(T_{r}\right)$ for any $r>0$. Let us define $b^{\prime}(x, \xi)$ by $b^{\prime}(x, \xi)=e(\xi) b(x, \xi)$ with the above $e(\xi)$. From (i) of Proposition 2.9 and (2.17) it follows that

$(2.17)^{\prime}\left|b^{\prime}(x, \xi)\right| \leqq C_{\varepsilon}^{\prime} \exp \left\{\sum_{j=1}^{n}\left(\lambda(|\operatorname{Im} y|)+\mu^{\prime}\left(\left|\operatorname{Re} \eta_{j}\right| / \operatorname{Im} \eta_{j}\right)+\varepsilon\right)\right.$ $\left.\times \operatorname{Im} \eta_{j}\right\}$,

where $\mu^{\prime}(t)=\mu(t)+C t^{2}$. We can prove a stronger result :

$(2.17)^{\prime \prime}\left|b^{\prime}(x, \xi)\right| \leqq C_{\varepsilon}^{\prime} \exp \left\{\sum_{j=1}^{n}\left(\lambda\left(C^{\prime}\left|\operatorname{Im} y_{\jmath}\right|\right)+\mu^{\prime}\left(\left|\operatorname{Re} \eta_{j}\right| / \operatorname{Im} \eta_{\jmath}\right)+\varepsilon\right)\right.$ $\left.\times \operatorname{Im} \eta_{j}\right\}$

with some $C^{\prime}>0$. In fact, if $\operatorname{Im} \eta_{j}>C \operatorname{Im} \eta_{k}$ for some $j$ and $k$, $(2.17)^{\prime \prime}$ is trivial because $b^{\prime}(x, \xi)$ is exponentially decreasing in such a region. On the other hand, if $\operatorname{Im} \eta_{j} \leqq C \operatorname{Im} \eta_{k}$ for any $j$ and $k$, we have $\sum_{j=1}^{n} \lambda(|\operatorname{Im} y|) \operatorname{Im} \eta_{j} \leqq \sum_{j=1}^{n} \lambda\left(C^{\prime}\left|\operatorname{Im} y_{j}\right|\right) \operatorname{Im} \eta_{j}$, thus (2.17)" holds. Thus we have $b^{\prime}(x, \xi) \in \mathcal{O}_{[\lambda],\left[\mu^{\prime}\right]}$, and we can define $\check{b}^{\prime}\left(x, x^{\prime}\right)$ by (2. 14). We define $\check{b}\left(x, x^{\prime}\right) d x^{\prime} \in \mathscr{L}_{\text {o }^{* *}}$ by $\breve{b}\left(x, x^{\prime}\right)=\check{b}^{\prime}\left(x, x^{\prime}\right)$. From (ii) of Proposition 2.9, it follows that this is well-defined. Using Proposition 2.8, we can prove that the mapping

$$
\mathscr{S}_{x^{*}} / \mathscr{N}_{0^{*}} \ni b(x, \xi) \longmapsto \breve{b}\left(x, x^{\prime}\right) d x^{\prime} \in \mathscr{L}_{x}
$$

is the inverse of (2.16), and thus (2.16) is an isomorphism. This proves Theorem 0.3.

\section{§ 3. Symbol Formula for Adjoint Operators}

In this section, we prove the symbol formula for the adjoint 
operator $A^{*} \in \mathscr{L}_{x^{\prime *}}$ of $\mathrm{A} \in \mathscr{L}_{x^{*} \text {. }}$ Let $\mathrm{A}=u\left(x, x^{\prime}\right) d x^{\prime}$. Let us assume for the moment that $u\left(x, x^{\prime}\right)$ satisfies the condition (1.1). Let $a>0$ be large enough, and define $y=y(x), \eta=\eta(\xi)$ by $(1.2),(1.4)$. It follows that there exist some scaling functions $\lambda(t), \mu(t)$, and some $r>0$

such that the defining function $v\left(x, x^{\prime}\right)$ of $u\left(x, x^{\prime}\right)$ belongs to $\mathcal{O}\left(W_{\lambda, \mu, r}^{(n)}\right)$. By definition we have $A^{*}=u\left(x^{\prime}, x\right) d x^{\prime}$, thus the defining function of $u\left(x^{\prime}, x\right)$ is $w\left(x, x^{\prime}\right)=v\left(x^{\prime}, x\right)$. Let $\lambda^{\prime}(t)=2 \lambda(2 t)$ and $\mu^{\prime}(t)=\mu(t) / 2$. We have the following

Lemma 3.1. If $\left(x, x^{\prime}\right) \in W_{\lambda^{\prime}, \mu^{\prime}, r / 2}^{(n)}$ and $0 \leqq \theta \leqq 1$, then we have $(x+$ $\left.\theta\left(x-x^{\prime}\right), x+(\theta-1)\left(x-x^{\prime}\right)\right) \in W_{\lambda, \mu, r}^{(n)}$.

Before the proof, we consider the meaning of Lemma 3.1. Let $\theta$ $=1$ in Lemma 3.1, then we have

$$
\left(x, x^{\prime}\right) \in W_{\lambda^{\prime}, \mu^{\prime}, r / 2}^{(n)} \quad \Longrightarrow \quad\left(2 x-x^{\prime}, x\right) \in W_{\lambda, \mu, r}^{(n)} .
$$

It follows that $w\left(x, 2 x-x^{\prime}\right)=v\left(2 x-x^{\prime}, x\right)$ as a function of $\left(x, x^{\prime}\right)$, belongs to $\mathcal{O}\left(W_{\lambda^{\prime}, \mu^{\prime}, r / 2}^{(n)}\right)$. This is important since we can calculate the Fourier transform of $w\left(x, x^{\prime}\right)$ from $w\left(x, 2 x-x^{\prime}\right)$ (considered at the point $\left.\stackrel{\circ}{x}^{\prime *}\right)$ as follows: The Fourier transform $\hat{w}(x, \xi)$ is defined by

$$
\hat{w}(x, \xi)=\int e^{-\left(x-x^{\prime}\right) \xi} w\left(x, x^{\prime}\right) d\left(x-x^{\prime}\right) \text {. }
$$

The precise definition of this integral is given just in the same way as in $\S 2$, but this time we are considering at the antipodal point $\stackrel{\circ}{x}^{*}$ of $\stackrel{\circ}{x}^{*}$. Thus every domain must be modified in an antipodal manner. If we write $x^{\prime \prime}=2 x-x^{\prime}$, we have

$$
\hat{w}(x, \xi)=\int e^{\left(x-x^{\prime \prime}\right) \xi} w\left(x, 2 x-x^{\prime \prime}\right) d\left(x-x^{\prime \prime}\right) .
$$

This time we regard $w\left(x, 2 x-x^{\prime \prime}\right)$ as an element of $\mathcal{O}\left(W_{\lambda^{\prime}, \mu^{\prime}, r^{\prime} / 2}^{(n)}\right)$, and we may regard $\hat{w}(x,-\xi)$ as the Fourier transform of $w\left(x, 2 x-x^{\prime \prime}\right)$. Replacing $x^{\prime \prime}$ by $x^{\prime}$, we have (3. 1) $\hat{w}(x,-\xi)=\int_{\Gamma} e^{-\left(x-x^{\prime}\right) \xi} w\left(x, 2 x-x^{\prime}\right) d\left(x-x^{\prime}\right)$.

Here the domain $\Gamma$ is the same as in (2.5).

To prove Lemma 3.1, we need the following

Lemma 3.2. If $\kappa(t)$ is a scaling function, we have 


$$
\kappa\left(t_{1}+t_{2}\right) \leqq \kappa\left(2 t_{1}\right)+\kappa\left(2 t_{2}\right), t_{1} t_{2} \geqq 0 .
$$

Proof. $\kappa\left(t_{1}+t_{2}\right) \leqq \kappa\left(2 \max \left(t_{1}, t_{2}\right)\right) \leqq \kappa\left(2 t_{1}\right)+\kappa\left(2 t_{2}\right)$. Q. E. D.

Proof of Lemma 3. 1. Let $\left(x, x^{\prime}\right) \in W_{\lambda^{\prime}, \mu^{\prime}, r / 2}^{(n)}$. Let $y_{j}=y_{j}(x), y_{j}{ }^{\prime}=y_{j}$ $\left(x^{\prime}\right)$, as before, and let $x(\theta)=x+\theta\left(x-x^{\prime}\right), x^{\prime}(\theta)=x+(\theta-1)\left(x-x^{\prime}\right)$, $y_{j}(\theta)=y_{j}(x(\theta))$, and $y_{j}^{\prime}(\theta)=y_{j}\left(x^{\prime}(\theta)\right)$.

We need to prove $\left(y_{j}(\theta), y_{j}^{\prime}(\theta)\right) \in W_{\lambda, \mu, r}, 1 \leqq j \leqq n$. Since $x(\theta)-x^{\prime}(\theta)$ $=x-x^{\prime}$, we have $y_{j}(\theta)-y_{j}{ }^{\prime}(\theta)=y_{j}-y_{j}{ }^{\prime}$, and we have $\left(y_{j}(\theta), y_{j}{ }^{\prime}(\theta)\right)$ $\in W_{r}, 1 \leqq j \leqq n(\operatorname{See}(2.1))$. Thus we only need to prove $I_{j}=\operatorname{Im}\left(y_{j}(\theta)\right.$ $\left.-y_{j}^{\prime}(\theta)\right)-\lambda\left(\left|\operatorname{Im} y_{j}(\theta)\right|\right)+\mu\left(\left|\operatorname{Re}\left(y_{j}(\theta)-y_{j}{ }^{\prime}(\theta)\right)\right|\right)>0,1 \leqq j \leqq n$. From Lemma 3.2, we obtain

$$
\begin{aligned}
I_{j}= & \operatorname{Im}\left(y_{j}-y_{,}{ }^{\prime}\right)-\lambda\left(\left|\operatorname{Im}\left(y_{j}+\theta\left(y_{j}-y_{j}{ }^{\prime}\right)\right)\right|\right)+\mu\left(\left|\operatorname{Re}\left(y_{j}-y_{j}{ }^{\prime}\right)\right|\right) \\
\geqq & \operatorname{Im}\left(y_{j}-y_{j}{ }^{\prime}\right)-\lambda\left(2\left|\operatorname{Im} y_{j}\right|\right)-\lambda\left(2 \theta\left|\operatorname{Im}\left(y_{j}-y_{j}{ }^{\prime}\right)\right|\right) \\
& \quad+\mu\left(\left|\operatorname{Re}\left(y_{j}-y_{j}{ }^{\prime}\right)\right|\right) .
\end{aligned}
$$

Taking $r>0$ small enough, we may assume that $\lambda(t) \leqq t / 4$ (See Remark (ii) after Definition 0.1). Thus we have

$$
\begin{aligned}
I_{j} \geqq & \operatorname{Im}\left(y_{j}-y_{j}^{\prime}\right)-\left|\operatorname{Im}\left(y_{j}-y_{j}^{\prime}\right)\right| / 2-\lambda\left(2\left|\operatorname{Im} y_{j}\right|\right) \\
& \quad+\mu\left(\left|\operatorname{Re}\left(y_{j}-y_{j}^{\prime}\right)\right|\right) \\
= & \min \left(3 \operatorname{Im}\left(y_{j}-y_{j}^{\prime}\right) / 2, \operatorname{Im}\left(y_{j}-y_{j}^{\prime}\right) / 2\right)-\lambda\left(2\left|\operatorname{Im} y_{j}\right|\right) \\
& \quad+\mu\left(\left|\operatorname{Re}\left(y_{j}-y_{j}^{\prime}\right)\right|\right) .
\end{aligned}
$$

Since $\left(x, x^{\prime}\right) \in W_{\lambda^{\prime}, \mu^{\prime}, r / 2}^{(n)}$ (i.e., each $\left.\left(y_{j}, y_{j}{ }^{\prime}\right) \in W_{\lambda^{\prime}, \mu^{\prime}, r / 2}\right)$, we have

$$
\begin{aligned}
& \min \left(3 \operatorname{Im}\left(y_{j}-y_{j}{ }^{\prime}\right) / 2, \operatorname{Im}\left(y_{j}-y_{j}{ }^{\prime}\right) / 2\right) \\
& >\lambda^{\prime}\left(\left|\operatorname{Im} y_{j}\right|\right) / 2-3 \mu^{\prime}\left(\operatorname{Re}\left(y_{j}-y_{j}^{\prime}\right) \mid\right) / 2 \\
& \geqq \lambda\left(2\left|\operatorname{Im} y_{j}\right|\right)-\mu\left(\left|\operatorname{Re}\left(y_{j}-y_{j}{ }^{\prime}\right)\right|\right),
\end{aligned}
$$

and thus $I_{j}>0,1 \leqq j \leqq n$.

Q.E. D.

Now we derive the asymptotic formula from (3.1). We write $\tilde{x}=x-x^{\prime}$, and $\tilde{a}\left(x, x^{\prime}\right)=a\left(x, x-x^{\prime}\right)$. If $N \in Z_{+}$, from (3.1) we obtain

$$
\begin{aligned}
\hat{w}(x,-\xi)= & \int_{\Gamma} e^{-\tilde{x} \xi} \tilde{v}(x+\tilde{x}, \tilde{x}) d \tilde{x} \\
= & \sum_{|\alpha| \leqq N-1} \frac{1}{\alpha !} \int_{\Gamma} e^{-\bar{x} \xi} \tilde{x}^{\alpha} \partial_{x}^{\alpha} \tilde{v}(x, \tilde{x}) d \tilde{x} \\
& \quad+\sum_{|\alpha|=N} \frac{N}{\alpha !} \int_{\Gamma} e^{-\bar{x} \xi} \tilde{x}^{\alpha}\left(\int_{0}^{1}(1-\theta)^{N-1} \partial_{x}^{\alpha} \tilde{v}(x+\theta \tilde{x}, \tilde{x}) d \theta\right) d \tilde{x}
\end{aligned}
$$

and thus 


$$
\hat{w}(x,-\xi)=\sum_{j=0}^{N-1} \hat{w}_{j}(x,-\xi)+\hat{R}_{N}(x,-\xi)
$$

Here

$$
\hat{w}_{j}(x,-\xi)=\sum_{|\alpha|=j} \frac{1}{\alpha !}(-1)^{|\alpha|} \partial_{x}^{\alpha} \partial_{\xi}^{\alpha} \hat{v}(x, \xi)
$$

and

$$
\hat{R}_{N}(x,-\xi)=\sum_{|\alpha|=N} \frac{N}{\alpha !}(-1)^{|\alpha|} \int_{0}^{1}(1-\theta)^{N-1} \partial_{x}^{\alpha} \partial_{\xi}^{\alpha} \hat{v}_{\theta}(x, \xi) d \theta
$$

where

$$
\hat{v}_{\theta}(x, \xi)=\int_{\Gamma} e^{-\tilde{x} \xi} \tilde{v}(x+\theta \tilde{x}, \tilde{x}) d \tilde{x} .
$$

Considering $\tilde{v}(x+\theta \tilde{x}, \tilde{x})=v\left(x+\theta\left(x-x^{\prime}\right), x+(\theta-1)\left(x-x^{\prime}\right)\right)$ as a function of $\left(x, x^{\prime}\right)$, we have $\tilde{v}(x+\theta \tilde{x}, \tilde{x}) \in \mathcal{O}\left(W_{\lambda^{\prime}, \mu^{\prime}, r / 2}^{(n)}\right)$, by virtue of Lemma 3.1. If $\mu^{\prime \prime}(t)=\tilde{\mu}^{\prime}(C t)$ and $0<r^{\prime} \ll r$, we have $\hat{v}_{\theta}(x, \xi) \in \mathcal{O}_{\lambda^{\prime}, \mu^{\prime \prime}}\left(T_{r^{\prime}}\right)$. From the proof of Proposition 2.5, we obtain a uniform estimate for $\hat{v}_{\theta}(x, \xi), 0 \leqq \theta \leqq 1$ : For any $\varepsilon>0$, there exists some $C_{\varepsilon}>0$ such that

(3.6) $\left|\hat{v}_{\theta}(x, \xi)\right| \leqq C_{\varepsilon} \exp \left\{\sum_{j=1}^{n}\left(\lambda^{\prime}\left(\left|\operatorname{Im} y_{j}\right|\right)+\mu^{\prime \prime}\left(\left|\operatorname{Re} \eta_{j}\right| / \operatorname{Im} \eta_{j}\right)+\varepsilon\right)\right.$

$$
\left.\times \operatorname{Im} \eta_{j}\right\}
$$

on $T_{r}, 0 \leqq \theta \leqq 1$. We can estimate the derivatives of $\hat{v}_{\theta}(x, \xi)$ as follows:

Proposition 3.3. Let $0<r^{\prime \prime} \ll r^{\prime}$, and let $0<r_{1}, r_{2}<r^{\prime \prime}$. For any $\varepsilon>0$ there exists some $C_{\varepsilon}>0$ such that

$$
\begin{aligned}
& \quad\left|\partial_{x}^{\alpha} \partial_{\xi}^{\alpha} \hat{v}_{\theta}(x, \xi)\right| \leqq C_{\varepsilon} \alpha ! \beta ! r_{1}^{-|\alpha|}\left(r_{2} \cdot \min _{1 \leqq i \leqq n} \operatorname{Im} \eta_{i}\right)^{-|\beta|} \\
& \times \exp \left\{\sum _ { j = 1 } ^ { n } \left(2 \lambda^{\prime}\left(2\left|\operatorname{Im} y_{j}\right|\right)+2 \mu^{\prime \prime}\left(4\left|\operatorname{Re} \eta_{j}\right| / \operatorname{Im} \eta_{j}\right)+\varepsilon\right.\right. \\
& \left.\left.\quad+2 \lambda^{\prime}\left(4 r_{1}\right)+2 \mu^{\prime \prime}\left(4 a r_{2}\right)\right) \operatorname{Im} \eta_{j}\right\}
\end{aligned}
$$

for $\alpha, \beta \in Z_{+}{ }^{n}, 0 \leqq \theta \leqq 1$, on $T_{r^{\prime \prime}}$.

Proof. Fix an arbitrary point $(x, \xi) \in T_{r^{\prime \prime}}$, and let $(\bar{x}, \bar{\xi}) \in C^{n} \times C^{n}$ be another point satisfying

(3.8) $\left|\bar{x}_{j}\right| \leqq r_{1}, \quad\left|\bar{\xi}_{j}\right| \leqq r_{2} \cdot \min _{1 \leqq i \leqq n} \operatorname{Im} \eta_{i}, 1 \leqq j \leqq n$.

Note that $\bar{x}$ does not denote the complex conjugate of $x$. We write as $\bar{y}_{j}=y_{j}(\bar{x}), \bar{\eta}_{j}=\eta_{j}(\bar{\xi}), 1 \leqq j \leqq n$. From (1.2) and (1.4) it is easy 
to see that if $r^{\prime \prime} \ll r^{\prime}$, then $(x+\bar{x}, \xi+\bar{\xi}) \in T_{r^{\prime}}$. From (3.6) it tollows that for any $\varepsilon>0$ there exists some $C_{\varepsilon}>0$ such that

$$
\begin{aligned}
& \left|\hat{v}_{\theta}(x+\bar{x}, \xi+\bar{\xi})\right| \leqq C_{\varepsilon} \exp \left\{\sum _ { j = 1 } ^ { n } \left(\lambda^{\prime}\left(\left|\operatorname{Im} y_{j}\right|+\left|\operatorname{Im} \bar{y}_{j}\right|\right)\right.\right. \\
& \left.\left.+\mu^{\prime \prime}\left(\left(\left|\operatorname{Re} \eta_{j}\right|+\left|\operatorname{Re} \bar{\eta}_{j}\right|\right) /\left(\operatorname{Im} \eta_{j}+\operatorname{Im} \bar{\eta}_{j}\right)\right)+\varepsilon\right)^{\prime}\left(\operatorname{Im} \eta_{j}+\operatorname{Im} \bar{\eta}_{j}\right)\right\} .
\end{aligned}
$$

Using Lemma 3.2 and (3.8), we obtain

$$
\begin{aligned}
& \left|\hat{v}_{\theta}(x+\bar{x}, \xi+\bar{\xi})\right| \leqq C_{\varepsilon} \exp \left\{\sum _ { j = 1 } ^ { n } \left(2 \lambda^{\prime}\left(2\left|\operatorname{Im} y_{j}\right|\right)+2 \lambda^{\prime}\left(4 r_{1}\right)\right.\right. \\
& \left.\left.+2 \mu^{\prime \prime}\left(4\left|\operatorname{Re} \eta_{j}\right| / \operatorname{Im} \eta_{j}\right)+2 \mu^{\prime \prime}\left(4 a r_{2}\right)+2 \varepsilon\right) \operatorname{Im} \eta_{j}\right\} .
\end{aligned}
$$

Using Cauchy integration theorem, we obtain Proposition 3.3.

Q.E.D.

Proposition 3. 4. Let $C_{0}>0$. We define $T(k) \subset C^{n} \times C^{n}, k \in Z_{+}, b \nu$

$$
\begin{aligned}
T(k)= & \left\{(x, \xi) \in C^{n} \times C^{n} ; C_{0}\left|x_{j}\right|<1,1 \leqq j \leqq n,\right. \\
& \operatorname{Im} \xi_{n}>C_{0}\left|\operatorname{Im} \xi_{j}\right|, 1 \leqq j \leqq n-1, \\
& \left.\operatorname{Im} \xi_{n}>C_{0}\left|\operatorname{Re} \xi_{j}\right|, 1 \leqq j \leqq n, \operatorname{Im} \xi_{n}>C_{0}(k+1)\right\} .
\end{aligned}
$$

Assume that $C_{0}$ and $C_{1}$ are large enough, and that $k \geqq|\alpha|,|\beta|$. Then for any $\varepsilon>0$ there exists some $C_{\varepsilon}>0$ such that

$$
\begin{aligned}
& \left|\partial_{x}^{\alpha} \partial_{\xi}{ }^{\beta} \hat{v}_{\theta}(x, \xi)\right| \leqq C_{\varepsilon} \alpha ! \beta ! \\
& \times \exp \left\{\left(\lambda\left(C_{1}|\operatorname{Im} x|\right)+\tilde{\mu}\left(C_{1}|\operatorname{Re} \xi| / \operatorname{Im} \xi_{n}\right)+\varepsilon\right) \operatorname{Im} \xi_{n}\right\} \\
& \times\left(C_{1} / \lambda^{-1}\left((|\alpha|+1) / \operatorname{Im} \xi_{n}\right)\right)^{|\alpha|}\left(C_{1} / \tilde{\mu}^{-1}\left((|\beta|+1) / \operatorname{Im} \xi_{n}\right) \operatorname{Im} \xi_{n}\right)^{|\beta|}
\end{aligned}
$$

on $T(k)$.

Proof. Let $(x, \xi) \in T(k), k \geqq|\alpha|,|\beta|$. If $C_{0}$ is large enough, then we can take $r_{1}=\lambda^{\prime-1}\left((|\alpha|+1) / \operatorname{Im} \xi_{n}\right) / 4, r_{2}=\mu^{\prime \prime}-1\left((|\beta|+1) / \operatorname{Im} \xi_{n}\right) /$ $4 a$ in Proposition 3.3 In fact, we have $r_{1} \leqq \lambda^{\prime-1}\left((|\alpha|+1) / \operatorname{Im} \xi_{n}\right)<$ $\lambda^{\prime-1}\left(1 / C_{0}\right)<r^{\prime \prime}$, if $C_{0}$ is large enough. Similarly, we have $r_{2}<r^{\prime \prime}$. From (3.7) it follows that for any $\varepsilon>0$ there exists some $C_{\varepsilon}>0$ such that (3. 10) $\left|\partial_{x}^{\alpha} \partial_{\xi}^{\beta} \hat{v}_{\theta}(x, \xi)\right| \leqq C_{\varepsilon} \alpha ! \beta !\left(4 / \lambda^{\prime-1}\left((|\alpha|+1) / \operatorname{Im} \quad \xi_{n}\right)\right)^{|\alpha|}$

$$
\begin{aligned}
& \times\left(4 a / \mu^{\prime \prime-1}\left((|\beta|+1) / \operatorname{Im} \xi_{n}\right) \min _{1 \leqq i \leqq n} \operatorname{Im} \eta_{i}\right)^{|\beta|} \\
& \times \exp \left\{\sum_{j=1}^{n}\left(2 \lambda^{\prime}\left(2\left|\operatorname{Im} y_{j}\right|\right)+2 \mu^{\prime \prime}\left(4\left|\operatorname{Re} \eta_{j}\right| / \operatorname{Im} \eta_{j}\right)+2 \varepsilon\right) \operatorname{Im} \eta_{j}\right\} \\
& \times \exp \left\{\sum_{j=1}^{n} 2(|\alpha|+|\beta|+2) \operatorname{Im} \eta_{j} / \operatorname{Im} \xi_{n}\right\} .
\end{aligned}
$$

If $C_{0}$ is large enough, from (1.4) and (1.5) we have

$$
2 n \cdot \min _{1 \leqq i \leqq n} \operatorname{Im} \eta_{i} \geqq \operatorname{Im} \xi_{n} \geqq \max _{1 \leqq i \leqq n} \operatorname{Im} \eta_{i}
$$

on $T(k)$. Substituting (3.11) into (3.10), we have 


$$
\begin{aligned}
& \left|\partial_{x}^{\alpha} \partial_{\xi}^{\beta} \hat{v}_{\theta}(x, \xi)\right| \leqq e^{4 n} C_{\varepsilon} \alpha ! \beta !\left(4 e^{2 n} / \lambda^{\prime-1}\left((|\alpha|+1) / \operatorname{Im} \xi_{n}\right)\right)^{|\alpha|} \\
& \times\left(8 n e^{2 n} a / \mu^{\prime \prime-1}\left((|\beta|+1) / \operatorname{Im} \xi_{n}\right) \operatorname{Im} \xi_{n}\right)|\beta| \\
& \times \exp \left\{\left(2 n \lambda^{\prime}\left(2 \max _{1 \leqq i \leqq n}\left|\operatorname{Im} y_{i}\right|\right)+2 n \mu^{\prime \prime}\left(8 n \max _{1 \leqq i \leqq n}\left|\operatorname{Re} \eta_{i}\right| / \operatorname{Im} \xi_{n}\right)\right.\right. \\
& \left.\quad+2 n \varepsilon) \operatorname{Im} \xi_{n}\right\} .
\end{aligned}
$$

From (0.2), (1.2), and (1.4), we have

(3.13) $\max _{1 \leqq i \leqq n}\left|\operatorname{Im} y_{i}\right| \leqq 2|\operatorname{Im} x|, \max _{1 \leqq i \leqq n}\left|\operatorname{Re} \eta_{i}\right| \leqq a|\operatorname{Re} \xi|$.

Substituting (3.13) into (3.12), we have

$$
\begin{aligned}
& \left|\partial_{x}{ }^{\alpha} \partial_{\xi}{ }_{\xi} \hat{v}_{\theta}(x, \xi)\right| \leqq e^{4 n} C_{\varepsilon} \alpha ! \beta !\left(4 e^{2 n} / \lambda^{\prime-1}\left((|\alpha|+1) / \operatorname{Im} \xi_{n}\right)\right)^{|\alpha|} \\
& \times\left(8 n e^{2 n} a / \mu^{\prime \prime-1}\left((|\beta|+1) / \operatorname{Im} \xi_{n}\right) \operatorname{Im} \xi_{n}\right)^{|\beta|} \\
& \times \exp \left\{\left(2 n \lambda^{\prime}(4|\operatorname{Im} x|)+2 n \mu^{\prime \prime}\left(8 n a|\operatorname{Re} \xi| / \operatorname{Im} \xi_{n}\right)+2 n \varepsilon\right) \operatorname{Im} \xi_{n}\right\} .
\end{aligned}
$$

From $(0.2)$, we have $\lambda^{\prime}(t)=2 \lambda(2 t) \leqq \lambda(4 t)$, and thus $\lambda^{\prime-1}(t) \geqq \lambda^{-1}(t) / 4$ 。 We have $\mu^{\prime \prime}(t)=\tilde{\mu}^{\prime}(C t)=\tilde{\mu}(2 C t) / 2 \leqq \tilde{\mu}(2 C t)$, and thus $\mu^{\prime \prime-1}(t) \geqq \tilde{\mu}^{-1}(t)$ $/ 2 C$. Furthermore, we have $2 n \lambda^{\prime}(t) \leqq \lambda^{\prime}(2 n t)$ and $2 n \mu^{\prime \prime}(t) \leqq \mu^{\prime \prime}(2 n t)$. Noting these facts, (3.9) follows directly from (3.14). Q.E.D.

Remark. (i) If $\theta=0$, we have $\hat{v}_{0}(x, \xi)=\hat{v}(x, \xi)$ (See $(3.5)$ ). Thus (3.9) gives an estimate also for $\hat{v}(x, \xi)$.

(ii) The estimate (3.9) will look more familiar if we let $\lambda(t)=$ $m_{1} t^{i}, \mu(t)=m_{2} t^{j}, m_{1}, m_{2}>0, i, j>1$. Then we have $\lambda^{-1}(t)=\left(t / m_{1}\right)^{1 / i}$, $\mu^{-1}(t)=\left(t / m_{2}\right)^{1 / j}$. Assume that $C_{\varepsilon}=C$ does not depend on $\varepsilon>0$, and let $\varepsilon \rightarrow+0, \theta=0$, in (3.9). If $(x, \xi) \in T(k) \cap \sqrt{-1} T^{*} M, k \geqq|\alpha|,|\beta|$, then we have

$$
\left|\partial_{x}^{\alpha} \partial_{\xi}^{\beta} \hat{v}(x, \xi)\right| \leqq C m_{1}{ }^{|\alpha| / i} m_{2}{ }^{\mid \beta ! / \jmath} \alpha !^{(i-1) / i} \beta !^{(j-1) / j}\left(\operatorname{Im} \xi_{n}\right)^{|\alpha| / i-|\beta|(J-1) / J},
$$

for $\alpha, \beta \in \mathbb{Z}_{+}{ }^{n}$. In this case we have $\hat{v}(x, \xi) \in S_{\rho, \delta}^{0}$ with $\rho=(j-1) / j$, $\delta=1 / i$. In this sense, our theory may be regarded as an analogue of $S_{\rho, \delta}^{m}$ theory, in the category of hyperfunctions. As was mentioned in $\S 0$, it seems inevitable to assume some condition, if one wants to obtain an asymptotic formula for adjoint operators (See §5).

Now we can give the asymptotic formula. Let $C_{0}, C_{1}{ }^{\prime}$ be large enough and assume that $\lambda(t)$ and $\tilde{\mu}(t)$ satisfy Condition $C_{0}, C_{1}^{\prime}$. We start from (3.2). If $(x, \xi) \in T(j)$, from (3.3) and (3.9) it follows that for any $\varepsilon>0$ there exists some $C_{\varepsilon}>0$ such that

$(3.15)\left|w_{j}(x,-\xi)\right|$ 


$$
\begin{aligned}
& \leqq 2^{n-1} C_{\varepsilon}\left(C_{1}^{2}(j+1) / \lambda^{-1}\left((j+1) / \operatorname{Im} \xi_{n}\right) \tilde{\mu}^{-1}\left((j+1) / \operatorname{Im} \xi_{n}\right) \operatorname{Im} \xi_{n}\right)^{j} \\
& \times \exp \left\{\left(\lambda\left(C_{1}|\operatorname{Im} x|\right)+\tilde{\mu}\left(C_{1}|\operatorname{Re} \xi| / \operatorname{Im} \xi_{n}\right)+\varepsilon\right) \operatorname{Im} \xi_{n}\right\} .
\end{aligned}
$$

If $C_{1}{ }^{\prime}>2 C_{1}{ }^{2}$, we have

$$
\begin{aligned}
& \left|w_{j}(x,-\xi)\right| \leqq 2^{n-j-1} C_{\varepsilon} \\
& \times \exp \left\{\left(\lambda\left(C_{1} \mid \operatorname{Im} x !\right)+\tilde{\mu}\left(C_{1}|\operatorname{Re} \xi| / \operatorname{Im} \xi_{n}\right)+\varepsilon\right) \operatorname{Im} \xi_{n}\right\} .
\end{aligned}
$$

The same estimate holds also for $\hat{R},(x,-\xi)$, on $T(j)$. A defining function of $u\left(x, x^{\prime}\right)$ (with $A^{*}=u\left(x^{\prime}, x\right) d x^{\prime}$ ) is given by (2.10) where $a(x, \xi)$ is replaced by $\hat{w}(x,-\xi)$, modulo microanalytic elements : i.e., as the defining function of $u\left(x^{\prime}, x\right)$, we may take

(3. 17) $w^{\prime}\left(x, x^{\prime}\right)=\frac{1}{(2 \pi \sqrt{-1})^{n}} \int_{\Delta} e^{-\left(x-x^{\prime}\right) \xi} w(x,-\xi) d \xi$.

The domain of this integral may be replaced by an arbitrary small conic neighborhood of $(0, \ldots, 0, \sqrt{-1})$ in $\sqrt{-1} \boldsymbol{R}^{n}$. We define $\Delta(j)$, $j \in \mathbb{Z}_{+}$, by

$$
\Delta(j)=\left\{\xi \in \sqrt{ }-\overline{\operatorname{lR}} \bar{R}^{n} ; \operatorname{Im} \xi_{n}>C_{0}\left|\operatorname{Im} \xi_{k}\right|, 1 \leqq k \leqq n-1, \operatorname{Im} \xi_{n}>C_{0}(j+1)\right\} .
$$

We replace $\Delta$ by $\Delta(0)$ in $(3.17)$. Then we have

$$
\text { (3. 18) } w^{\prime}\left(x, x^{\prime}\right) \equiv \frac{1}{(2 \pi \sqrt{-1})^{n}} \int_{\Delta(0)} e^{-\left(x-x^{\prime}\right) \xi} \hat{w}(x,-\xi) d \xi
$$

modulo microanalytic elements. From (3.2) and (3.16) we have

$$
\begin{aligned}
w^{\prime}\left(x, x^{\prime}\right) \equiv & \frac{1}{(2 \pi \sqrt{-1})^{n}}\left\{\sum_{j=0}^{N-1} \int_{\Delta(\jmath+1)} e^{-\left(x-x^{\prime}\right) \xi} \hat{w}_{j}(x,-\xi) d \xi\right. \\
& +\sum_{j=0}^{N-1} \int_{\Delta(j) \backslash \Delta(j+1)} e^{-\left(x-x^{\prime}\right) \xi} \hat{R},(x,-\xi) d \xi \\
& \left.+\int_{\Delta(N)} e^{-\left(x-x^{\prime}\right) \xi} \hat{R}_{N}(x,-\xi) d \xi\right\}
\end{aligned}
$$

by induction on $N$. By (3.16) for $\hat{w}_{j}(x,-\xi)$ and $\hat{R}_{j}(x,-\xi)$, we can let $N \rightarrow+\infty$, and thus we have

$$
\begin{gathered}
w^{\prime}\left(x, x^{\prime}\right) \equiv \frac{1}{(2 \pi \sqrt{-1})^{n}}\left\{\sum_{j=0}^{\infty} \int_{\Delta(j+1)} e^{-\left(x-x^{\prime}\right) \xi} \hat{w}_{j}(x,-\xi) d \xi\right. \\
\left.\quad+\sum_{j=0}^{\infty} \int_{\Delta(j) \backslash \Delta(j+1)} e^{-\left(x-x^{\prime}\right) \xi} \hat{R},(x,-\xi) d \xi\right\} .
\end{gathered}
$$

The second term gives an microanalytic element, and we have

$$
w\left(x, x^{\prime}\right) \equiv \frac{1}{(2 \pi \sqrt{-1})^{n}} \sum_{j=0}^{\infty} \int_{\Delta(j+1)} e^{-\left(x-x^{\prime}\right) \xi} \hat{w}_{j}(x,-\xi) d \xi .
$$


It is easy to see that the right-hand side is convergent, and it is the defining function of $u\left(x^{\prime}, x\right)$.

Proof of Theorem 0.5. We use the notation of Theorem 0.5. Let $A=u\left(x, x^{\prime}\right) d x^{\prime} \in \mathscr{L}_{{ }^{\circ},}$, and let $\sigma(A) \in \mathscr{S}_{\lambda, \mu}$. Thus there exists some $C$ $>0$, and for any $\varepsilon>0$ there exists some $C_{\varepsilon}>0$ such that

$$
\begin{aligned}
& |\sigma(A)(x, \xi)| \leqq C_{\varepsilon} \exp \left\{\sum_{j=1}^{n}\left(\lambda(C|\operatorname{Im} y|)+\mu\left(C\left|\operatorname{Re} \eta_{j}\right| / \operatorname{Im} \eta_{j}\right)+\varepsilon\right)\right. \\
& \left.\times \operatorname{Im} \eta_{j}\right\}
\end{aligned}
$$

on $T_{r}$, with some $r>0$ (We have chosen some $y$ and $\eta$ ). For the sake of simplicity, we assume a stronger estimate

$$
\text { (3. 19)' } \begin{aligned}
& |\sigma(A)(x, \xi)| \leqq C_{\varepsilon} \exp \left\{\sum_{j=1}^{n}\left(\lambda\left(C\left|\operatorname{Im} y_{j}\right|\right)+\mu\left(C\left|\operatorname{Re} \eta_{j}\right| / \operatorname{Im} \eta_{j}\right)+\varepsilon\right)\right. \\
& \left.\times \operatorname{Im} \eta_{j}\right\}
\end{aligned}
$$

on $T_{r}$, with the same letter $\lambda$ and $\mu$, and that $\lambda, \mu$ satisfy Condition $C_{0}, C_{1}$. Then the above argument applies for $\sigma\left(A^{*}\right)(x,-\xi)$, and we obtain Theorem 0.5. In the general case (under the weaker assumption (3.19)), we can also follow the above argument, although several points require more careful consideration. We do not give the proof for the general case, because it is essentially the same thing.

Q.E.D.

\section{§4. Symbol Formula for Composite Operators}

Let $A_{i}=u_{i}\left(x, x^{\prime}\right) d x^{\prime} \in \mathscr{L}_{x^{*}}, i=1,2$. In this section we give an asymptotic representation of the symbol of

$$
A_{1} A_{2}=\left(\int u_{1}\left(x, x^{\prime \prime}\right) u_{2}\left(x^{\prime \prime}, x^{\prime}\right) d x^{\prime \prime}\right) d x^{\prime} \in \mathscr{L}_{x^{\circ *}} .
$$

We assume that $u_{i}\left(x, x^{\prime}\right), i=1,2$, satisfy the condition (1.1), for the moment. Let $a>0$ be large enough, and define $y=y(x)$ by (1.2). It follows that the corresponding defining functions $v_{i}\left(x, x^{\prime}\right), i=1,2$, are holomorphic on $W_{i_{i}^{\prime}, \mu_{i}, r}^{(n)}$, with some $r>0$, and some scaling functions $\lambda_{i}(t), \mu_{i}(t)$, respectively.

We first investigate the domain of definition of the function $\left(v_{1} * v_{2}\right)\left(x, x^{\prime}\right)=\int v_{1}\left(x, x^{\prime \prime}\right) v_{2}\left(x^{\prime \prime}, x^{\prime}\right) d x^{\prime \prime}$. We define new scaling functions $\lambda(t)$ and $\mu(t)$ by 
(4. 1) $\lambda(t)=C \lambda_{1}(2 t)+C \lambda_{2}(2 t)$,

(4.2) $\mu(t)=\frac{1}{C} \min _{0 \leqq \theta \leqq 1}\left(\mu_{1}(\theta t)+\mu_{2}((1-\theta) t)\right)$,

where $C>0$ is a large constant. Then we have the following

Lemma. 4.1. $\lambda(t)$ and $\mu(t)$ are scaling functions.

Proof. We only need to prove (0.2) for $\mu(t)$, other properties being trivial. We denote as $\mu_{0}(t)=\mu(t) / t, \mu_{i 0}(t)=\mu_{i}(t) / t, i=1,2$. If $t<t^{\prime}$, then we have

$$
\begin{aligned}
\mu_{0}(t) & =\frac{1}{C} \min _{0 \leqq \theta \leqq 1}\left(\mu_{1}(\theta t) / t+\mu_{2}((1-\theta) t) / t\right) \\
& =\frac{1}{C} \min _{0 \leqq \theta \leqq 1}\left(\theta \mu_{10}(\theta t)+(1-\theta) \mu_{20}((1-\theta) t)\right) \\
& <\frac{1}{C} \min _{0 \leqq \theta \leqq 1}\left(\theta \mu_{10}\left(\theta t^{\prime}\right)+(1-\theta) \mu_{20}\left((1-\theta) t^{\prime}\right)\right)=\mu_{0}\left(t^{\prime}\right) .
\end{aligned}
$$

Q.E. D.

We next show that $\left(v_{1} * v_{2}\right) \quad\left(x, x^{\prime}\right) \in \mathcal{O}\left(W_{\lambda, \mu, r^{\prime}}^{(n)}\right)$ with $0<r^{\prime} \ll r$. Precisely speaking $v_{1} * v_{2}$ is defined by

$$
\left(v_{1} * v_{2}\right) \quad\left(x, x^{\prime}\right)=\int_{\Gamma} v_{1}\left(x, x^{\prime \prime}\right) v_{2}\left(x^{\prime \prime}, x^{\prime}\right) d\left(x-x^{\prime \prime}\right) .
$$

Here $\Gamma$ is the same as in (2.5): $\Gamma=\left\{x-x^{\prime \prime} \in C^{n} ; y_{3}(x)-y_{j}\left(x^{\prime \prime}\right) \in \gamma\right.$, $1 \leqq j \leqq n\}$, and $\gamma$ is a curve from $s_{1}$ to $s_{2}$. We had better define $s_{1}$ and $s_{2}$ explicitly. Without loss of generality we may choose $s_{1}=$ $-\mu_{1}^{-1}\left(2 r^{\prime}\right)-\sqrt{-1} r^{\prime}$ and $s_{2}=\mu_{1}^{-1}\left(2 r^{\prime}\right)-\sqrt{-1} r^{\prime}$. Note that if $\left|\operatorname{Im} y_{j}\right|$ $\left\langle\lambda_{1}^{-1}\left(r^{\prime}\right), 1 \leqq j \leqq n\right.$, then we have $\operatorname{Im} s_{i}>\lambda_{1}\left(\left|\operatorname{Im} y_{j}(x)\right|\right)-\mu_{1}\left(\left|\operatorname{Re} s_{i}\right|\right)$, $i=1,2,1 \leqq j \leqq n$. If $G>0$ is large, we have $\operatorname{Im} s_{i}<-\frac{2}{G} \mu_{1}\left(\left|\operatorname{Re} s_{i}\right|\right)$, $i=1$, 2. As in $\S 2$, for any $\varepsilon>0$ we can choose a curve $\gamma=\gamma_{\varepsilon}$ from $s_{1}$ to $s_{2}$ such that if $\left|\operatorname{Im} y_{j}(x)\right|<\lambda_{1}^{-1}\left(r^{\prime}\right), y_{j}(x)-y,\left(x^{\prime \prime}\right) \in \gamma_{\varepsilon}, 1 \leqq j \leqq n$, then we have $\left(x, x^{\prime \prime}\right) \in W_{\lambda_{1}, \mu_{1}, r}^{(n)}$ and

$$
\begin{aligned}
\operatorname{Im}\left(y_{j}(x)\right. & \left.-y_{j}\left(x^{\prime \prime}\right)\right)<\lambda_{1}\left(\left|\operatorname{Im} y_{j}\right|\right) \\
& -\frac{2}{C} \mu_{1}\left(\left|\operatorname{Re}\left(y_{j}(x)-y_{j}\left(x^{\prime \prime}\right)\right)\right|\right)+\varepsilon, 1 \leqq j \leqq n .
\end{aligned}
$$

From now on, we write as $y_{j}^{\prime \prime}=y_{j}\left(x^{\prime \prime}\right)$, as before. We define $W_{\lambda, \mu, r, r^{\prime}}^{(n)}$ 
$\in \mathbb{C}^{n} \times \mathbb{C}^{n}$ by

$$
\begin{aligned}
W_{\lambda, \mu, r, r^{\prime}}^{(n)}= & \left\{\left(x, x^{\prime}\right) \in \mathbb{C}^{n} \times \mathbb{C}^{n} ;\left|\operatorname{Re} y_{j}\right|<r / 2,\left|\operatorname{Im} y_{j}\right|<\lambda^{-1}\left(r^{\prime}\right),\right. \\
& \left|\operatorname{Re}\left(y_{j}-y_{j}{ }^{\prime}\right)\right|<r / 2,\left|\operatorname{Im}\left(y_{j}-y_{j}^{\prime}\right)\right|<\lambda^{-1}\left(r^{\prime}\right), \\
& \operatorname{Im}\left(y_{j}-y_{j}^{\prime}\right)>\lambda\left(\left|\operatorname{Im} y_{j}\right|\right)-\mu\left(\mid \operatorname{Re}\left(y_{j}-y_{j}^{\prime}\right) !\right), \\
& 1 \leqq j \leqq n\} .
\end{aligned}
$$

We have $W_{\lambda, \mu, r^{\prime}}^{(n)}=\left\{\left(x, x^{\prime}\right) \in W_{\lambda, \mu, r, r^{\prime}}^{(n)} ;\left|\operatorname{Re} \quad y_{j}\right|<r^{\prime}, \quad\left|\operatorname{Im} \quad y_{j}\right|<r^{\prime}\right.$, $\left.\left|\operatorname{Re}\left(y_{j}-y_{j}{ }^{\prime}\right)\right|<r^{\prime},\left|\operatorname{Im}\left(y_{j}-y_{j}{ }^{\prime}\right)\right|<r^{\prime}, 1 \leqq j \leqq n\right\}$, and thus $W_{\lambda, \mu, r, r^{\prime}}^{(n)}$ and $W_{\lambda, \mu, r^{\prime}}^{(n)}$ are essentially of the same property. We have the following

Lemma 4. 2. $\quad\left(v_{1} * v_{2}\right)\left(x, x^{\prime}\right) \in \mathcal{O}\left(W_{\lambda, \mu, r, r^{\prime}}^{(n)}\right)\left(\subset \mathcal{O}\left(W_{\lambda, \mu, r^{\prime}}^{(n)}\right)\right)$.

Proof. Let $\left(x, x^{\prime}\right) \in W_{\lambda, \mu, r, r}^{(n)}$ There exists some $\varepsilon>0$ such that $\operatorname{Im}\left(y_{j}-y_{j}{ }^{\prime}\right)>\lambda\left(\left|\operatorname{Im} y_{j}\right|\right)-\mu\left(\left|\operatorname{Re}\left(y_{j}-y_{j}{ }^{\prime}\right)\right|\right)+2 \varepsilon, 1 \leqq j \leqq n$. We need to prove that if $y_{j}-y_{j}^{\prime \prime} \in \gamma_{\varepsilon}, 1 \leqq j \leqq n$, then $\left(x, x^{\prime \prime}\right) \in W_{\lambda_{1}, \mu_{1}, r}^{(n)}$, and $\left(x^{\prime \prime}, x^{\prime}\right)$ $\in W_{\lambda_{2}, \mu_{2}, r}^{(n)}$. We only need to prove

(4.6) $\quad I_{j}=\operatorname{Im}\left(y_{j}^{\prime \prime}-y_{j}^{\prime}\right)-\lambda_{2}\left(\left|\operatorname{Im~} y_{j}^{\prime \prime}\right|\right)+\mu_{2}\left(\left|\operatorname{Re}\left(y_{j}^{\prime \prime}-y_{j}^{\prime}\right)\right|\right)>0$,

$1 \leqq j \leqq n$, other properties being trivially satisfied. Let us prove (4.6). Using Lemma 3.2, we obtain

$$
\begin{aligned}
I_{j} & \geqq \operatorname{Im}\left(y_{j}-y_{j}{ }^{\prime}\right)-\operatorname{Im}\left(y_{j}-y_{j}^{\prime \prime}\right)-\lambda_{2}\left(2\left|\operatorname{Im} y_{j}\right|\right) \\
& -\lambda_{2}\left(2\left|\operatorname{Im}\left(y_{j}-y_{j}^{\prime \prime}\right)\right|\right)+\mu_{2}\left(\left|\operatorname{Re}\left(y_{j}^{\prime \prime}-y_{j}{ }^{\prime}\right)\right|\right) .
\end{aligned}
$$

We may assume that $\lambda_{2}(t) \leqq t / 4$, and thus we have

$$
\begin{aligned}
& I_{j} \geqq \operatorname{Im}\left(y_{j}-y_{j}{ }^{\prime}\right)-\operatorname{Im}\left(y_{j}-y_{j}^{\prime \prime}\right)-\lambda_{2}\left(2\left|\operatorname{Im} y_{j}\right|\right) \\
& \quad-\left|\operatorname{Im}\left(y_{j}-y_{j}^{\prime \prime}\right)\right| / 2+\mu_{2}\left(\left|\operatorname{Re}\left(y_{j}^{\prime \prime}-y_{j}^{\prime}\right)\right|\right) \\
& =\operatorname{Im}\left(y_{j}-y_{j}{ }^{\prime}\right)-\max \left(3 \operatorname{Im}\left(y_{j}-y_{j}^{\prime \prime}\right) / 2, \operatorname{Im}\left(y_{j}-y_{j}^{\prime \prime}\right) / 2\right) \\
& \quad-\lambda_{2}\left(2\left|\operatorname{Im} y_{j}\right|\right)+\mu_{2}\left(\left|\operatorname{Re}\left(y_{j}^{\prime \prime}-y_{j}^{\prime}\right)\right|\right) .
\end{aligned}
$$

From (4.4) and (4.5) we have

$$
\begin{aligned}
I_{j}> & \left\{\lambda\left(\left|\operatorname{Im} y_{j}\right|\right)-\mu\left(\left|\operatorname{Re}\left(y_{j}-y_{j}{ }^{\prime}\right)\right|\right)+2 \varepsilon\right\} \\
& -\left\{\frac{3}{2} \lambda_{1}\left(\left|\operatorname{Im} y_{j}\right|\right)-\frac{1}{G} \mu_{1}\left(\left|\operatorname{Re}\left(y_{j}-y_{j}^{\prime \prime}\right)\right|\right)+3 \varepsilon / 2\right\} \\
& -\lambda_{2}\left(2\left|\operatorname{Im} y_{j}\right|\right)+\mu_{2}\left(\left|\operatorname{Re}\left(y_{j}^{\prime \prime}-y_{j}^{\prime}\right)\right|\right)
\end{aligned}
$$

Using (4.1) and (4.2) we have $I_{j}>0$. 
From Lemma 4.2 it follows that we can calculate $\left(v_{1} * v_{2}\right)^{\wedge}(x, \xi)$ according to $\S 2$, the domain of integration being appropriately chosen. Let $s_{1}{ }^{\prime}, s_{2}{ }^{\prime}$ be as in $\S 2$. We define them explicitly by

$$
s_{1}{ }^{\prime}=-\mu^{-1}\left(6 r^{\prime}\right)-\sqrt{-1} r^{\prime}, s_{2}{ }^{\prime}=\mu^{-1}\left(6 r^{\prime}\right)-\sqrt{-1} r^{\prime} .
$$

Let $\gamma^{\prime}$ be a path from $s_{1}{ }^{\prime}$ to $s_{2}{ }^{\prime}$. We have

$$
\begin{aligned}
& \left(v_{1} * v_{2}\right)^{\wedge}(x, \xi) \\
& \quad=\int_{\Gamma^{\prime}} \int_{\Gamma} e^{-\left(x-x^{\prime}\right) \xi_{1}}\left(x, x^{\prime \prime}\right) v_{2}\left(x^{\prime \prime}, x^{\prime}\right) d\left(x-x^{\prime \prime}\right) d\left(x-x^{\prime}\right) \\
& =\int_{\Gamma} \int_{\Gamma^{\prime}} e^{-\left(x-x^{\prime}\right) \xi} v_{1}\left(x, x^{\prime \prime}\right) v_{2}\left(x^{\prime \prime}, x^{\prime}\right) d\left(x-x^{\prime}\right) d\left(x-x^{\prime \prime}\right) .
\end{aligned}
$$

Here $\Gamma$ is as before, and $\Gamma^{\prime}=\left\{x-x^{\prime} \in \mathbb{C}^{n} ; y_{0}-y_{j}{ }^{\prime} \in \gamma^{\prime}, 1 \leqq j \leqq n\right\}$. We note that if

$$
\left|\operatorname{Im} y_{j}\right|<\lambda^{-1}\left(r^{\prime} / 2\right), \quad\left|\operatorname{Re} y_{j}\right|<r / 2, \quad 1 \leqq j \leqq n,
$$

then we have $\operatorname{Im} s_{i}{ }^{\prime}>\lambda\left(\left|\operatorname{Im} y_{j}\right|\right)-\mu\left(\mid \operatorname{Re} s_{i}{ }^{\prime}\right), i=1,2,1 \leqq j \leqq n$. Let $C^{\prime}$ be large enough. Then we have $\operatorname{Im} s_{i}{ }^{\prime}<-\frac{2}{C^{\prime}} \mu\left(\left|\operatorname{Re} s_{i}{ }^{\prime}\right|\right)$, $i=1$, 2. As before, for any $\varepsilon>0$ we can choose $\gamma^{\prime}=\gamma_{\varepsilon}^{\prime}$ such that if $x \in C^{n}$ satisfies (4.9) and $y_{0}-y^{\prime}{ }^{\prime} \in \gamma_{\varepsilon}{ }^{\prime}, 1 \leqq j \leqq n$, then we have $\left(x, x^{\prime}\right)$ $\in W_{\lambda, \mu, r, r^{\prime}}^{(n)}$ and

(4. 10) $\operatorname{Im}\left(y_{j}-y_{j}{ }^{\prime}\right)<\lambda(\mid \operatorname{Im} y, i)-\frac{1}{C^{\prime}} \mu\left(\left|\operatorname{Re}\left(y_{j}-y_{j}{ }^{\prime}\right)\right|\right)+\varepsilon, 1 \leqq j \leqq n$ 。

Note that (4.8) is well-defined if $x \in C^{n}$ satisfies (4.9).

To give an asymptotic representation from (4.8), we need to replace the domain of integration $\Gamma^{\prime}$ in (4.8) by another one. We fix an arbitrary point $\left(x, x^{\prime \prime}\right) \in C^{n} \times C^{n}$ such that (4.9) is satisfied and $x-x^{\prime \prime} \in \Gamma$. Let $\gamma^{\prime \prime}$ be a path from $s_{1}{ }^{\prime}+\left(y_{j}-y_{0}^{\prime \prime}\right)$ to $s_{2}{ }^{\prime}+\left(y_{j}-y_{j}^{\prime \prime}\right)$, and let $\Gamma^{\prime \prime}=\Gamma^{\prime \prime}\left(x, x^{\prime \prime}\right)=\left\{x-x^{\prime} \in \mathbb{C}^{n} ; y_{j}-y_{j}^{\prime} \in \gamma^{\prime \prime}, 1 \leqq j \leqq n\right\}$. We define $\hat{h}(x, \xi)$ by

$$
\hat{h}(x, \xi)=\int_{\Gamma} \int_{\Gamma^{\prime \prime}} e^{-\left(x-x^{\prime}\right) \xi_{1}}\left(x, x^{\prime \prime}\right) v_{2}\left(x^{\prime \prime}, x^{\prime}\right) d\left(x-x^{\prime}\right) d\left(x-x^{\prime \prime}\right) .
$$

Then we have the following

Lemma 4. 3. If $x \in \mathbb{C}^{n}$ satisfies (4.9), then $\hat{h}(x, \xi)$ is well-defined, and we have $\hat{h}(x, \xi)-\left(v_{1} * v_{2}\right)^{\wedge}(x, \xi) \in \mathscr{N}_{x}$. 
Proof. The difference of (4.8) and (4.11) is the following: In (4.8) each $y_{j}-y_{j}{ }^{\prime}$ moves on $\gamma^{\prime}$, but in (4.11) moves on $\gamma^{\prime \prime}$. The difference of these two paths is $l_{1 j} \cup l_{2 j}$, where $l_{i j}, i=1,2,1 \leqq j \leqq n$, is the line segment between $s_{i}{ }^{\prime}$ and $s_{i}{ }^{\prime}+\left(y_{j}-y_{j}{ }^{\prime \prime}\right)$ (See figure 4). Let $J_{1}, J_{2} \subset\{1,2, \ldots, n\}$ be such that $J_{1} \cup J_{2}=\{1,2, \ldots, n\}$ is a disjoint union, and let $J=\left(J_{1}, J_{2}\right)$ be a pair of such $J_{1}$ and $J_{2}$. We define $\Gamma^{\prime}{ }_{J}$ by $\Gamma^{\prime}{ }_{J}=\left\{x-x^{\prime} \in C^{n} ; y_{j}-y_{j}{ }^{\prime} \in \gamma^{\prime}, j \in J_{1}, y_{j}-y_{j}{ }^{\prime} \in l_{1 j} \cup l_{2 j}\right.$, $\left.j \in J_{2}\right\}$. Then $\hat{h}(x, \xi)-\left(v_{1} * v_{2}\right)^{\wedge}(x, \xi)$ is a finite sum of several integrals of the following form, with $J_{2} \neq \phi$ :

$$
\int_{\Gamma} \int_{\Gamma^{\prime}{ }_{J}} e^{-\left(x-x^{\prime}\right) \xi_{1}} v_{1}\left(x, x^{\prime \prime}\right) v_{2}\left(x^{\prime \prime}, x^{\prime}\right) d\left(x-x^{\prime}\right) d\left(x-x^{\prime \prime}\right) .
$$

Let $I_{j}, 1 \leqq j \leqq n$, be as in (4.6). We shall show that if $x-x^{\prime} \in \Gamma^{\prime}{ }_{J}$, then $I_{j}>0,1 \leqq j \leqq n$, and

$$
\operatorname{Im}\left(y_{j}-y_{j}{ }^{\prime}\right)<-r^{\prime} / 4, j \in J_{2} \text {. }
$$

Note that $I_{j}>0,1 \leqq j \leqq n$, means $\left(x^{\prime \prime}, x^{\prime}\right)$ belongs to the domain of definition of $v_{2}\left(x^{\prime \prime}, x^{\prime}\right)$, and thus (4.12) and $\hat{h}(x, \xi)$ is well-defined. Furthermore, (4.13) means

$$
\left|e^{-\left(y_{j}-y_{j}{ }^{\prime} \eta_{j}\right.}\right| \leqq e^{-r^{\prime} \operatorname{Im} \eta_{j} / 4+! \operatorname{Re} \eta_{j} \mid}, j \in J_{2}
$$

and it follows easily that if $J_{2} \neq \phi$, then (4. 12) belongs to $\bigoplus_{k} \partial_{k} \mathcal{O}_{[\lambda],[\mu]}$

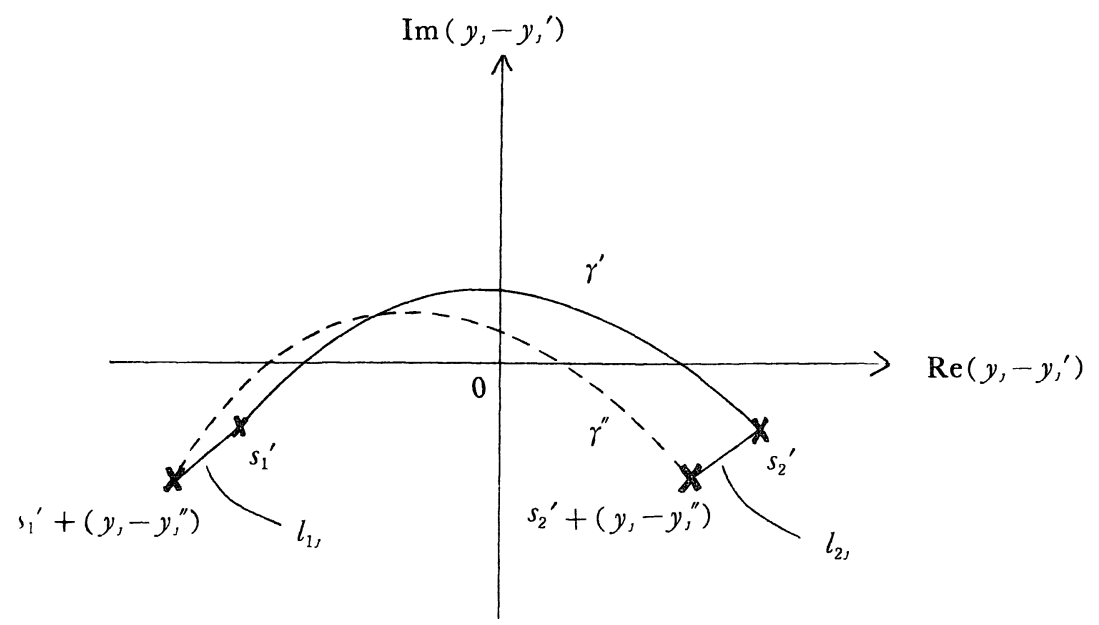

Figure 4 
$\subset \mathscr{N}_{x^{*}}$, and the above two facts give the lemma.

Now we prove $I_{j}>0,1 \leqq j \leqq n$. If $j \in J_{1}$, the proof is the same as Lemma 4.2, and let $j \in J_{2}$. We denote $y_{j}-y_{0}{ }^{\prime} \in l_{i}$, by $y_{0}-y_{j}{ }^{\prime}=s_{i}{ }^{\prime}+$ $(1-\theta)\left(y_{j}-y_{j}^{\prime \prime}\right), 0 \leqq \theta \leqq 1$. From Lemma 3.2, we have

$$
\begin{aligned}
I_{j}= & \operatorname{Im} s_{i}{ }^{\prime}-\theta \operatorname{Im}\left(y_{j}-y_{j}{ }^{\prime \prime}\right)-\lambda_{2}\left(\left|\operatorname{Im} y_{j}{ }^{\prime}\right|\right)+\mu_{2}\left(\left|\operatorname{Re}\left(y_{j}{ }^{\prime \prime}-y_{j}{ }^{\prime}\right)\right|\right) \\
\geqq & \operatorname{Im} s_{i}{ }^{\prime}-\theta \operatorname{Im}\left(y_{j}-y_{j}^{\prime \prime}\right)-\lambda_{2}\left(2\left|\operatorname{Im} y_{j}\right|\right) \\
& \quad-\lambda_{2}\left(2\left|\operatorname{Im}\left(y_{j}-y_{j}{ }^{\prime}\right)\right|\right)+\mu_{2}\left(\left|\operatorname{Re}\left(y_{j}^{\prime \prime}-y_{j}{ }^{\prime}\right)\right|\right) .
\end{aligned}
$$

We may assume that $\lambda_{2}(t) \leqq t / 4$, and thus

$$
\begin{aligned}
I_{j} \geqq & \operatorname{Im} s_{i}{ }^{\prime}-\theta \operatorname{Im}\left(y_{j}-y_{j}{ }^{\prime \prime}\right)-\lambda_{2}\left(2\left|\operatorname{Im} y_{j}\right|\right) \\
& \quad-\left|\operatorname{Im}\left(y_{j}-y_{j}{ }^{\prime \prime}\right)\right| / 2+\mu_{2}\left(\left|\operatorname{Re}\left(y_{j}{ }^{\prime \prime}-y_{j}{ }^{\prime}\right)\right|\right) \\
\geqq & \operatorname{Im} s_{i}{ }^{\prime}+\min \left(-3 \operatorname{Im}\left(y_{j}-y_{j}^{\prime \prime}\right) / 2, \operatorname{Im}\left(y_{j}-y_{j}^{\prime \prime}\right) / 2\right) \\
& \quad-\lambda_{2}\left(2\left|\operatorname{Im} y_{j}\right|\right)+\mu_{2}\left(\left|\operatorname{Re}\left(y_{j}{ }^{\prime \prime}-y_{j}{ }^{\prime}\right)\right|\right) .
\end{aligned}
$$

We may have chosen the path $\gamma_{\varepsilon}$ (for the variable $y_{j}-y_{j}^{\prime \prime}$ ) in such a way that we have $\operatorname{Im}\left(y_{j}-y_{j}{ }^{\prime \prime}\right) \geqq \operatorname{Im} s_{i}\left(=-r^{\prime}=\operatorname{Im} s_{i}{ }^{\prime}\right)$ on $\gamma_{\varepsilon}$ (See figure 3 ). Thus we have

$$
\begin{aligned}
I_{j} \geqq & 2 \operatorname{Im} s_{i}{ }^{\prime}+\min \left(-5 \operatorname{Im}\left(y_{j}-y_{j}^{\prime \prime}\right) / 2,-\operatorname{Im}\left(y,-y_{j}^{\prime \prime}\right) / 2\right) \\
& -\lambda_{2}\left(2\left|\operatorname{Im} y_{j}\right|\right)+\mu_{2}\left(\left|\operatorname{Re}\left(y_{j}^{\prime \prime}-y_{j}{ }^{\prime}\right)\right|\right) .
\end{aligned}
$$

From (4.1), (4.2), and (4.4), it follows that

$$
\begin{aligned}
I_{j} \geqq & 2 \operatorname{Im} s_{i}{ }^{\prime}+\left\{-5 \lambda_{1}\left(\left|\operatorname{Im} y_{j}\right|\right) / 2+\mu_{1}\left(\left|\operatorname{Re}\left(y_{j}-y_{j}{ }^{\prime \prime}\right)\right|\right) / C-5 \varepsilon / 2\right\} \\
& -\lambda_{2}\left(2\left|\operatorname{Im} y_{j}\right|\right)+\mu_{2}\left(\left|\operatorname{Re}\left(y_{j}{ }^{\prime \prime}-y_{j}{ }^{\prime}\right)\right|\right) \\
\geqq & 2 \operatorname{Im} s_{i}{ }^{\prime}-\lambda\left(\left|\operatorname{Im} y_{j}\right|\right)+\mu\left(\left|\operatorname{Re}\left(y_{j}-y_{j}{ }^{\prime \prime}\right)\right|+\left|\operatorname{Re}\left(y_{j}{ }^{\prime \prime}-y_{j}{ }^{\prime}\right)\right|\right) \\
& -5 \varepsilon / 2 .
\end{aligned}
$$

We have $\left|\operatorname{Re}\left(y_{j}-y_{j}^{\prime \prime}\right)\right|+\left|\operatorname{Re}\left(y_{j}^{\prime \prime}-y_{j}^{\prime}\right)\right|=\left|\operatorname{Re}\left(y_{j}-y_{j}^{\prime \prime}\right)\right|+$ $\left|\operatorname{Re} s_{i}{ }^{\prime}-\theta \operatorname{Re}\left(y_{j}-y_{j}{ }^{\prime \prime}\right)\right| \geqq\left|\operatorname{Re} s_{i}{ }^{\prime}\right|$, and thus

$$
I_{j} \geqq 2 \operatorname{Im} s_{i}{ }^{\prime}-\lambda\left(\left|\operatorname{Im} y_{j}\right|\right)+\mu\left(\left|\operatorname{Re} s_{i}{ }^{\prime}\right|\right)-5 \varepsilon / 2>3 r^{\prime}-5 \varepsilon / 2 \text {. }
$$

We can take $\varepsilon<r^{\prime}$ and thus $I_{j}>0,1 \leqq j \leqq n$.

We next prove (4.13). From (4.4) and (4.9) we have

$$
\begin{aligned}
\operatorname{Im}\left(y_{j}-y_{j}{ }^{\prime}\right) & =\operatorname{Im} s_{i}{ }^{\prime}+(1-\theta) \operatorname{Im}\left(y_{j}-y_{j}{ }^{\prime \prime}\right) \\
& <\operatorname{Im} s_{i}{ }^{\prime}+(1-\theta)\left(\lambda_{1}\left(\left|\operatorname{Im} y_{j}\right|\right)+\varepsilon\right)<-r^{\prime} / 2+\varepsilon .
\end{aligned}
$$

We can take $\varepsilon<r^{\prime} / 4$, and thus $\operatorname{Im}\left(y_{j}-y_{j}{ }^{\prime}\right)<-r^{\prime} / 4$.

Q. E. D.

Let $\gamma^{\prime}$ be some path from $s_{1}{ }^{\prime}$ to $s_{2}{ }^{\prime}$ again, and let $\Gamma^{\prime}=\left\{x^{\prime \prime}-x^{\prime}\right.$; 
$\left.\in \mathbb{C}^{n} ; y_{j}^{\prime \prime}-y_{j}{ }^{\prime} \in \gamma^{\prime}, 1 \leqq j \leqq n\right\}$. Since $x-x^{\prime} \in \Gamma^{\prime \prime} \Leftrightarrow x^{\prime \prime}-x^{\prime} \in \Gamma^{\prime}$, we have (4.11)' $\hat{h}(x, \xi)=\iint_{\Gamma} e_{\Gamma^{\prime}} e^{-\left(x-x^{\prime}\right) \xi} v_{1}\left(x, x^{\prime \prime}\right) v_{2}\left(x^{\prime \prime}, x^{\prime}\right) d\left(x^{\prime \prime}-x^{\prime}\right) d\left(x-x^{\prime}\right)$.

Now we can proceed as in $\S 3$. We write as $\tilde{x}^{\prime}=x^{\prime \prime}-x^{\prime}, \tilde{x}^{\prime \prime}=x-x^{\prime \prime}$, and

$$
\tilde{v}_{1}\left(x, x^{\prime \prime}\right)=v_{1}\left(x, x-x^{\prime \prime}\right), \tilde{v}_{2}\left(x^{\prime \prime}, x^{\prime}\right)=v_{2}\left(x^{\prime \prime}, x^{\prime \prime}-x^{\prime}\right) 。
$$

From (4.11)' we have

$$
\begin{aligned}
\hat{h}(x, \xi)= & \int_{\Gamma} \int_{\Gamma^{\prime}} e^{-\left(\tilde{z}^{\prime}+\tilde{z}^{\prime \prime}\right) \xi} \tilde{v}_{1}\left(x, \tilde{x}^{\prime \prime}\right) \tilde{v}_{2}\left(x-\tilde{x}^{\prime \prime}, \tilde{x}^{\prime}\right) d \tilde{x}^{\prime} d \tilde{x}^{\prime \prime} \\
= & \sum_{|\alpha| \leq N-1} \frac{1}{\alpha !} \int_{\Gamma} \int_{\Gamma^{\prime}} e^{-\left(\tilde{x}^{\prime}+\tilde{x}^{\prime \prime}\right) \xi} \tilde{v}_{1}\left(x, \tilde{x}^{\prime \prime}\right) \\
+ & \times\left(-\tilde{x}^{\prime \prime}\right)^{\alpha} \partial_{x}^{\alpha} \tilde{v}_{2}(x, \tilde{x}) d \tilde{x}^{\prime} d \tilde{x}^{\prime \prime} \\
\alpha ! & \int_{\Gamma} \int_{\Gamma^{\prime}} e^{-\left(\tilde{x}^{\prime}+\tilde{x}^{\prime \prime}\right) \xi} \tilde{v}_{1}\left(x, \tilde{x}^{\prime \prime}\right)\left(-\tilde{x}^{\prime \prime}\right)^{\alpha} \\
& \times \int_{0}^{1}(1-\theta)^{N-1} \partial_{x}^{\alpha} \tilde{v}_{2}\left(x-\theta \tilde{x}^{\prime \prime}, \tilde{x}^{\prime}\right) d \theta \\
& \times d \tilde{x}^{\prime} d \tilde{x}^{\prime \prime} .
\end{aligned}
$$

It follows that

$$
\text { (4.14) } \hat{h}(x, \xi)=\sum_{j=0}^{N-1} \hat{h}_{j}(x, \xi)+S_{N}(x, \xi)
$$

Here

$$
\text { (4.15) } \quad \hat{h},(x, \xi)=\sum_{|\alpha|=j} \frac{1}{\alpha !} \partial_{\xi}^{\alpha} \hat{v}_{1}(x, \xi) \partial_{x}^{\alpha} \hat{v}_{2}(x, \xi),
$$

and

$$
\text { (4.16) } S_{N}(x, \xi)=\sum_{\mid \alpha i=N} \frac{N}{\alpha !} \int_{0}^{1}(1-\theta)^{N-1} \hat{h}_{\theta, \alpha}(x, \xi) d \theta
$$

where

(4.17) $\quad \hat{h}_{\theta, \alpha}(x, \xi)=\int_{\Gamma} \int_{\Gamma^{\prime}} \partial_{\tilde{\xi}}^{\alpha}\left(e^{-z^{\prime \prime} \xi}\right) e^{-\bar{x}^{\prime} \xi} \tilde{v}_{1}\left(x, \tilde{x}^{\prime \prime}\right) \partial_{x}^{\alpha} \tilde{v}_{2}\left(x-\theta \tilde{x}^{\prime \prime}, \tilde{x}^{\prime}\right) d \tilde{x}^{\prime} d \tilde{x}^{\prime \prime}$.

We can estimate these derivatives as in $\S 3$, and obtain the following : Let $C_{0}, C_{1}$ be large enough, and let $r^{\prime \prime}>0$ be small enough。 Let $T(j) \subset \mathbb{C}^{n} \times \mathbb{C}^{y_{n}}, j \in \mathbb{Z}_{+}$, be the domains defined in Proposition 3.4. Then for any $\varepsilon>0$ there exists some $C_{\varepsilon}>0$ such that

(4. 18) $\left|\mathscr{K}_{j}(x, \xi)\right| \leqq C_{\varepsilon} \exp \left\{\sum_{j=1}^{n}\left(\lambda\left(C_{1}|\operatorname{Im} x|\right)+\tilde{\mu}\left(C_{1}|\operatorname{Re} \xi| / \operatorname{Im} \xi_{n}\right)+\varepsilon\right)\right.$ 


$$
\begin{aligned}
& \times \operatorname{Im} \xi_{n}\left(C_{1}(j+1) / \tilde{\mu}_{1}^{-1}(j+1) / \operatorname{Im} \xi_{n}\right) \lambda_{2}^{-1} \\
& \left.\left.\quad\left((j+1) / \operatorname{Im} \xi_{n}\right) \operatorname{Im} \xi_{n}\right)^{j}\right\}
\end{aligned}
$$

on $T(j)$. The same estimate holds also for each $S_{\jmath}(x, \xi)$ on $T(j)$.

Now we assume that $\tilde{\mu}_{1}(t)$ and $\lambda_{2}(t)$ satisfy Condition $C_{0}, C_{1}{ }^{\prime}$ where $G_{1}{ }^{\prime} \geqq 2 C_{1}$. Since we have $\left(v_{1} * v_{2}\right)^{\wedge}(x, \xi) \equiv \hat{h}(x, \xi)$ modulo $\mathscr{N}_{x}$, we have

$$
\left(v_{1} * v^{2}\right)\left(x, x^{\prime}\right) \equiv \frac{1}{(2 \pi \sqrt{-1})^{n}} \int_{\Delta(0)} e^{\left(x-x^{\prime}\right) \xi} \hat{h}(x, \xi) d \xi
$$

neglecting microanalytic elements Here we define $\Delta(j), j \in \mathbb{Z}_{+}$, by $\Delta(j)=\left\{\xi \in \sqrt{-1} \mathbb{R}^{n} ; \operatorname{Im} \xi_{n}>C_{0}\left|\operatorname{Im} \xi_{k}\right|, 1 \leqq k \leqq n-1, \operatorname{Im} \xi_{n}>C_{0} j\right\}$, as in $\S 3$. As was shown in $\S 3$, from $(4.14)-(4.19)$ we obtain

$$
\left(v_{1} * v_{2}\right) \quad\left(x, x^{\prime}\right) \equiv \frac{1}{(2 \pi \sqrt{-1})^{n}} \sum_{j=0}^{\infty} \int_{\Delta(\jmath+1)} e^{\left(x-x^{\prime}\right) \xi} \hat{h_{\jmath}}(x, \xi) d \xi,
$$

modulo microanalytic elements. The right-hand side is convergent, and it becomes the defining function of the kernel function $\int u_{1}\left(x, x^{\prime \prime}\right)$ $u_{2}\left(x^{\prime \prime}, x^{\prime}\right) d x^{\prime \prime}$ of $A_{1} A_{2}$ 。

We obtain Theorem 0.6 from the above argument, by the same reasoning as Proof of Theorem 0.5 in $\S 3$.

\section{§5. Two Examples}

In this section we give two important examples, and also explain about the notion of analytic $S_{\rho, \delta}^{m}$-class, defined by [9].

We first consider the Lewy-Mizohata type operators $P_{ \pm}(x, D)=$ $D_{1} \pm \sqrt{-1} x_{1} D_{2}$ at $\stackrel{\circ}{x}=(0 ; 0, \sqrt{-1}) \in \sqrt{-1} S^{*} \mathbb{R}^{2}$. Let us calculate the symbol function $E(x, \xi)$ of the right inverse $E(x, D) \in \mathscr{L}_{x^{* *}}$ of $P_{-}(x, D)$. $E(x, \xi)$ should satisfy

$$
\left(\xi_{1}-\sqrt{-1} x_{1} \xi_{2}\right) E(x, \xi)+\partial_{x_{1}} E(x, \xi)=1 .
$$

We define $\stackrel{\circ}{x}_{1}=\stackrel{\circ}{x}_{1}(\xi) \in \mathbb{C}$ by $\stackrel{\circ}{x}_{1}=\sqrt{-1}(\sqrt{2}-1) \xi_{1} / \xi_{2}$, and $\varphi\left(x_{1}, t, \xi\right)$ by $\varphi\left(x_{1}, t, \xi\right)=\frac{\sqrt{-1}}{2} x_{1}{ }^{2} \xi_{2}-x_{1} \xi_{1}-\frac{\sqrt{-1}}{2} t^{2} \xi_{2}+t \xi_{1}$. If $t$ lies on the line segment between $\stackrel{\circ}{x}_{1}$ and $x_{1}$, then we have 
$\operatorname{Re} \varphi\left(x_{1}, t, \xi\right) \leqq C\left(|\operatorname{Im} x|^{2}+\left(|\operatorname{Re} \xi| / \operatorname{Im} \xi_{2}\right)^{2}\right) \operatorname{Im} \xi_{2}$ with some $G>0$. We define $E(x, \xi)$ by

$$
E(x, \xi)=\int_{\dot{x}_{1}}^{x_{1}} \exp \varphi\left(x_{1}, t, \xi\right) d t .
$$

Then it follows that $E(x, \xi) \in \mathscr{S}_{\text {o }^{*}}$ and that $E(x, \xi)$ satisfies (5.1). The corresponding operator $E(x, D)$ thus satisfies

$$
P_{-}(x, D) E(x, D)=I d \text { 。 }
$$

Now let us calculate the symbol function of $E(x, D) P_{-}(x, D)$. It is given by

$$
\begin{aligned}
& \left(\xi_{1}-\sqrt{-1} x_{1} \xi_{2}\right) E\left(x_{1}, \xi\right)-\sqrt{-1} \xi_{2} \partial_{\xi_{1}} E(x, \xi) \\
& =\exp \varphi\left(x_{1}, x_{1}, \xi\right)-\sqrt{2} \exp \varphi\left(x_{1}, \stackrel{\circ}{1}_{1}, \xi\right) \\
& =1-K(x, \xi),
\end{aligned}
$$

where

$$
K(x, \xi)=\sqrt{2} \exp \left\{\frac{\sqrt{-1}}{2} x_{1}{ }^{2} \xi_{2}+\frac{\sqrt{-1}}{2} \xi_{1}{ }^{2} \xi_{2}{ }^{-1}-x_{1} \xi_{1}\right\} \in \mathscr{S}_{\dot{x}^{*}}
$$

Denoting the corresponding operator by $K(x, D)$, we obtain

$$
E(x, D) P_{-}(x, D)=I d-K(x, D) .
$$

As for $P_{+}(x, D)$, we define $F(x, \xi)$ by

$$
F(x, \xi)=\int_{\xi_{1}}^{\xi_{1}} \exp \phi\left(x_{1}, \xi, \tau\right) d \tau
$$

where $\quad \stackrel{\circ}{\xi}_{1}=\sqrt{-1}(\sqrt{2}-1) x_{1} \xi_{2}$ and $\quad \phi\left(x_{1}, \quad \xi, \quad \tau\right)=\frac{\sqrt{-1}}{2} \xi_{1}^{2} \hat{\xi}_{2}^{-1}-x_{1} \xi_{1}$ $-\frac{\sqrt{-1}}{2} \tau^{2} \xi_{2}^{-1}+x_{1} \tau$. Then we have $F(x, \xi) \in \mathscr{S}_{\mathrm{x}^{*}}$ and

$$
P_{+}(x, D) F(x, D)=I d-K(x, D), F(x, D) P_{+}(x, D)=I d,
$$

for the corresponding operator $F(x, D)$.

The next example is the parametrix of the Grusin type operator

$$
P(x, D)=D_{1}^{2}+x_{1}^{2} D_{2}^{2}+A(x, D),
$$

where $A(x, D)$ is a microdifferential operator of order 1 defined at $\stackrel{\circ}{x}^{*}=(0 ; 0, \sqrt{-1}) \in \sqrt{-1} S^{*} \mathbb{R}^{2}$ as before. Now we give the definition of analytic $S_{\rho, \delta}^{m}$-class. This class was first defined by G. Métivier [9], but here we give a slightly modified definition: 
Definition 5.1. Let $r, m \in \boldsymbol{R}$, and let $0<\boldsymbol{\rho} \leqq 1,0 \leqq \delta<1$. We say that a $C^{\infty}$ function $a(x, \xi)$ defined on

$$
\left\{(x, \xi) \in \mathbb{R}^{n} \times \sqrt{-1} \boldsymbol{R}^{n} ;|x|<r,|\operatorname{Im} \xi,|<r \operatorname{Im} \xi_{n}, 1 \leqq j \leqq n-1\right\}
$$

belongs to the analytic $S_{\rho, \delta}^{m}$-class if

$$
\begin{aligned}
\left|\partial_{x}^{\alpha} \partial_{\xi}^{\beta} a(x, \xi)\right| & \leqq C^{|\alpha|+|\beta|+1}|\xi|^{m}\left((|\alpha|+1)+(|\alpha|+1)^{1-\delta}|\xi|^{\delta}\right)^{|\alpha|} \\
& \times\left((|\beta|+1)|\xi|^{-1}+(|\beta|+1)^{\rho}|\xi|^{-\rho}\right)^{|\beta|}
\end{aligned}
$$

with some $G>0$ on (5.2), for any $\alpha, \beta \in \boldsymbol{Z}_{+}{ }^{n}$.

Let $0<\rho, \delta<1$, let $\lambda(t)=C_{1} t^{\delta}, \mu(t)=C_{2} t^{1 /(1-\rho)}$, and let $a(x, \xi) \in \mathscr{S}_{\dot{x}^{*}}$ satisfy

$$
\left.|a(x, \xi)| \leqq C_{3}|\xi|^{m} \exp \left\{\lambda(\operatorname{Im} x \mid)+\mu\left(|\operatorname{Re} \xi| / \operatorname{Im} \xi_{n}\right)\right) \operatorname{Im} \xi_{n}\right\}
$$

on a conical complex neighbohood of ${ }^{*} *(0 ; 0, \ldots, 0, \sqrt{-1}) \in \sqrt{-1} T^{*}$ $\boldsymbol{R}^{n}$. Let $C$ be large and let $r$ be small. From Proposition 3.4 it is easy to see that if $(x, \xi)$ belongs to the domain (5.2) and satisfies

$$
r \operatorname{Im} \xi_{n}>|\alpha|+1,|\beta|+1,
$$

then $a(x, \xi)$ satisfies (5.3). Even if $(x, \xi)$ does not satisfy (5.5), we can also prove similarly that $a(x, \xi)$ satisfies (5.3) on (5.2). Thus (5.4) means that $a(x, \xi)$ belongs to the analytic $S_{\rho, \delta}^{m}$-class. Conversely assume that $a(x, \xi)$ belongs to the analytic $S_{\rho, \delta}^{m}$-class. Considering the Taylor expansion around real points, it follows that $a(x, \xi)$ satisfies $(5.4)$ on a conical complex neighborhood of ${ }^{\circ}$. The analytic $S_{\rho, \delta}^{m}$-classes are the most typical examples of our symbol theory. Our theory is more general in two points: It contains operators of infinite orders, and it involves more complicated scaling functions.

For the Grusin type operators, Métivier proved (essentially) the following: Let $C$ be large and let $r$ be small. Assume that the principal symbol $A_{1}(x, \xi)$ of $A(x, D)$ satisfies

$$
A_{1}(x, \xi) \xi_{2}^{-1} \oplus\{-(2 j+1) \sqrt{-1} ; j=0,1,2, \ldots\} .
$$

Then we can obtain some $G_{j}(x, \xi), j \in \boldsymbol{Z}_{+}$, of analytic $S_{1 / 2,1 / 2}^{C-j / 2}$-class respectively, which satisfies

$$
\begin{aligned}
\left|\partial_{x}{ }^{\alpha} \partial_{\xi}^{\beta} G_{j}(x, \xi)\right| & \leqq C^{j+|\alpha|+|\beta|+1} j^{1 / 2}|\xi|^{C-j / 2} \\
& \times\left.\left((|\alpha|+1)+(|\alpha|+1)^{1 / 2}|\xi|^{1 / 2}\right)\right|^{\alpha} \mid \\
& \times\left((|\beta|+1)|\xi|^{-1}+(|\beta|+1)^{1 / 2}|\xi|^{-1 / 2}\right)^{|\beta|}
\end{aligned}
$$


on (5.2), such that $\sum_{j=0}^{\infty} G_{j}(x, \xi)$ gives the parametrix of $P(x, D)$. More precisely, let $C_{0}$ be large enough, and consider the following power series:

$$
L\left(x, x^{\prime}\right)=\sum_{j=0}^{\infty} \frac{1}{\left(2 \pi \sqrt{-1)^{2}}\right.} \int_{\Delta^{\prime}(j)} e^{\left(x-x^{\prime}\right) \xi} G_{j}(x, \xi) d \xi .
$$

Here we have defined

$$
\Delta^{\prime}(j)=\left\{\xi \in \sqrt{-1 R^{2}} ; \operatorname{Im} \xi_{2}>C_{0}\left|\operatorname{Im} \xi_{1}\right|, C_{0}(j+1)\right\} 。
$$

We can prove that $L\left(x, x^{\prime}\right)$ is well-defined and that $G(x, D)$ $=L\left(x, x^{\prime}\right) d x^{\prime}$ is a microlocal operator defined at ${ }^{\circ} *$, which satisfies

$$
P(x, D) G(x, D)=G(x, D) P(x, D)=I d .
$$

Each symbol $G_{j}(x, \xi)$ is obtained successively by solving a transport equation. See [9] and [10] for details.

\section{Bibliography}

[1] Aoki, T., Symbols and formal symbols of pseudodifferential operators, Advanced studies in Pure Mathematics, 4 (1984), 181-208.

[2] Hörmander, L., Pseudo-differential operators and hypoelliptic equations, Proc. Symposium on Singular Integrals, Amer. Math. Soc., 10 (1967), 138-183.

[3] Kashiwara, M. and Kawai, T., Pseudo-differential operators in the theory of hyperfunctions, Proc. Japan Acad., 46 (1970), 1130-1134.

[4] Kashiwara, M. and Scapira, P., Problème de Cauchy pour les systèmes microdifférentiels dans le domaine complexe, Inventiones Math., 46 (1978), 17-38.

[5] Kataoka, K., On the theory of Radon transformations of hyperfunctions, J. Fac. Sci. Univ. Tokyo, Sect. IA Math., 28 (1981), 331-413.

[6] Komatsu, H., A local version of Bochner's tube theorem, J. Fac. Sci. Univ. Tokyo, Sect. IA Math., 19 (1972), 201-214.

[ 7 ] Kumanogo, H., Pseudo-differential operators and the uniqueness of the Cauchy problem, Comm. Pure and Appl. Math., 22 (1969), 73-129.

[8] Melin, A. and Sjöstrand, J., Fourier integral operators with complex valued phase functions, Lect. Notes in Math., 459, Springer, 1975, 120-223.

[9] Métivier, G., Analytic hypoellipticity for operators with multiple characteristics, Comm. Part. Diff. Eq., 6 (1981), 1-90.

[10] Okaji, T., Analytic hypoellipticity for operators with symplectic characteristics, J. Math. Kyoto Univ., 25 (1985), 489-514.

[11] Sato, M., Kawai, T., and Kashiwara, M., Microfunctions and pseudo-differential equations, Lect. Notes in Math., 287, Springer, 1973, 265-529. 